Portland State University

PDXScholar

Spring 6-20-2013

\title{
The Use of Music as a Pedagogical Tool in Higher Education Sociology Courses: Faculty Member Perspectives and Potential Barriers
}

Jerry C.L. Loveless

Portland State University

Follow this and additional works at: https://pdxscholar.library.pdx.edu/open_access_etds

Part of the Educational Methods Commons, and the Educational Sociology Commons Let us know how access to this document benefits you.

Recommended Citation

Loveless, Jerry C.L., "The Use of Music as a Pedagogical Tool in Higher Education Sociology Courses: Faculty Member Perspectives and Potential Barriers" (2013). Dissertations and Theses. Paper 1100. https://doi.org/10.15760/etd.1100

This Thesis is brought to you for free and open access. It has been accepted for inclusion in Dissertations and Theses by an authorized administrator of PDXScholar. Please contact us if we can make this document more accessible: pdxscholar@pdx.edu. 
The Use of Music as a Pedagogical Tool in Higher Education Sociology Courses:

Faculty Member Perspectives and Potential Barriers

by

Jerry C.L. Loveless

A thesis submitted in partial fulfillment of the requirements for the degree of

Master of Science

in Sociology

Thesis Committee:

Peter J. Collier, Chair

Martha Balshem

Randy Blazak

Portland State University

2013 
(C) 2013 Jerry C.L. Loveless 


\begin{abstract}
Previous research has identified student engagement as an important antecedent to student learning in higher education. Although student engagement is viewed as important for learning, a significant number of college students still report frequently feeling bored in their courses. The use of music as a pedagogical tool is believed to be beneficial for promoting student engagement and student learning in higher education sociology courses, yet it has been suggested that sociology faculty members do not commonly incorporate the technique into their courses. The purpose of this comparative interview study is to explore higher education sociology faculty members' understandings of the use of music as a pedagogical tool, and the perceived importance of student engagement to student learning among higher education sociology faculty members.

In this study, it is found that higher education sociology faculty members believe student engagement can lead to increased student learning. It is also found that higher education sociology faculty members generally identify music as an effective pedagogical tool for promoting student engagement and learning in higher education sociology courses. Interestingly, participants believed the use of music as a pedagogical tool to be an uncommon practice in higher education sociology courses in the United States. As part of their efforts to explain their choices to use or not use music as a pedagogical tool, faculty participants described potential barriers that may impact faculty member choices to use music in their higher education sociology courses.
\end{abstract}


Sociology faculty participants in this study agreed that a lack of discussion of pedagogical tools among colleagues and in teaching courses might serve as a potential barrier for the use of music as a pedagogical tool. Higher education sociology faculty participants also identified a lack of knowledge of how to use music as a pedagogical tool as a potential barrier for the use of music in sociology courses. This research suggests that the lack of faculty knowledge of music as a pedagogical tool may be due to the lack of discussion of pedagogical tools both among colleagues and in the teaching courses completed by higher education sociology faculty members.

Past research has suggested that sociology faculty members need to create an environment that encourages students to be active and engaged participants in their own learning through building a community of learners. This study suggests that higher education sociology faculty members may successfully build a community of learners through using music as a pedagogical tool in their courses. This study recommends that changes at the departmental level need to occur in order to make it easier for sociology faculty members to gain the knowledge required to use music effectively in their courses. Suggestions for practice and future research are provided. 


\section{Dedication}

For those who challenge the potential "iron cage" of bureaucratic structures with creativity, innovation, and art. 


\section{Acknowledgments}

First and foremost, I would like to thank all my friends and family for their ongoing support during this life transition. I am especially indebted to my Mom and Stepdad Byron, Aunt Sue, Uncle John, Cousins Sarah and Bobby, the Hinchen Family, Molly Day, Heather Guevara, Lisa Baumann, and fellow musicians Stuart Smith, Liam Mullaney, Jon Rydman, Keith Walker and Wade Newman IV for helping me stay sane throughout this academic endeavor.

Second, I would like give high praise to the members of my thesis committee for their on-going guidance and amazing intellect. Specifically, I would like to thank Dr. Randy Blazak for the awesome inquisitions, Dr. Martha Balshem for the numerous insightful and jovial conversations (go Party Committee go!), and my thesis chair Dr. Peter Collier for being a constant maestro at providing feedback and helping me see "the big picture."

Finally, I would like to thank the Federal Student Aid Program, the Oregon TUI Program, and the Portland State University Sociology Department for their financial support. Without their assistance I would have not had the monetary backing needed to complete this program. 


\section{Table of Contents}

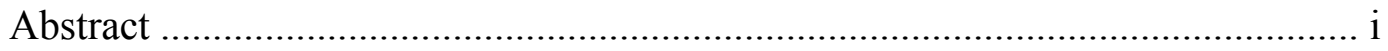

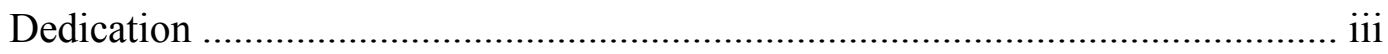

Acknowledgements ............................................................................. iv

List of Tables ....................................................................................... vii

List of Figures .................................................................................... viii

Chapter One

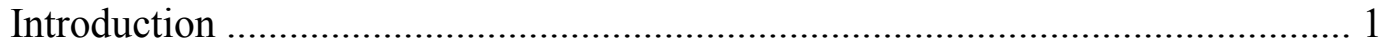

Chapter Two

Review of Literature ........................................................................... 10

Chapter Three

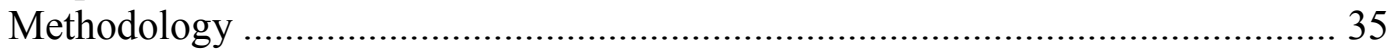

Chapter Four

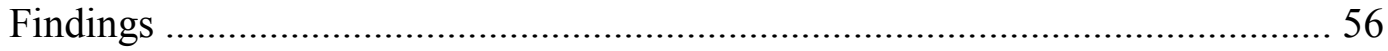

Chapter Five

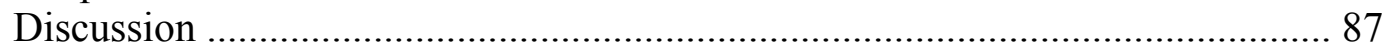

Chapter Six

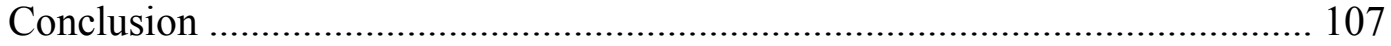

References ....................................................................................... 123

Appendices

A: Recruitment Email I ............................................................ 129

B: Recruitment Email II .......................................................... 130

C: Recruitment Telephone Script I ............................................. 131

D: Recruitment Telephone Script II ............................................. 132 
E: Informed Consent Document ................................................... 133

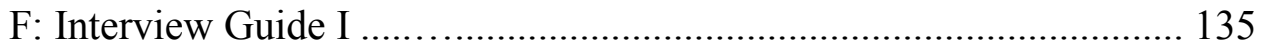

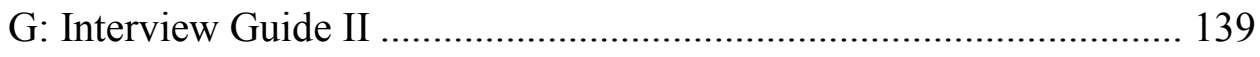

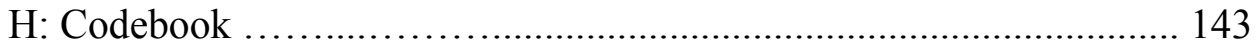

I: Figure of Potential Barriers to Inform Future Research .................. 145

J: Human Subjects Approval ....................................................... 146 


\section{List of Tables}

Table 1: Participant Demographic Characteristics ....................................... 40

Table 2: Participant Graduate School Characteristics ................................ 42 


\section{List of Figures}

Figure 1: Similarities and Differences Between Faculty Groups; Student Engagement, Student Learning, and Pedagogical Tools

Figure 2: Similarities and Differences Between Faculty Groups; Benefits and Costs of Using Music as a Pedagogical Tool

Figure 3: Similarities and Differences Between Faculty Groups; Potential

Structural Barriers Associated With Using Music as a Pedagogical Tool 71 


\section{Chapter One: Introduction}

Do higher education sociology faculty members and departments view student engagement as an important precursor to learning? While recent studies suggested student engagement is indeed important for learning outcomes, there has been a lack of literature discussing specifically whether sociology faculty members believe this to be true. Do higher education sociology faculty members and departments view the use of music as a legitimate pedagogical tool? While past studies in sociology and other disciplines have suggested college faculty members hold a relatively negative view of the use of music as a pedagogical tool, there is a lack of literature focusing on whether or not sociology faculty members today view the use of music as a pedagogical tool positively or negatively.

The purpose of this research is to explore higher education sociology members' understandings of the use of music as a pedagogical tool ${ }^{1}$ and the perceived importance of student engagement in relation to student learning. Music as a pedagogical tool will be generally defined as the use of recorded music and song lyrics in the higher education sociology classroom to help transmit sociological knowledge. Student engagement will be generally defined as a 'student's psychological investment in learning, comprehending

\footnotetext{
1 Drawing from Bernstein's discussion on how "pedagogy defines what counts as valid transmission of knowledge" (Sadovnik 1991:51), pedagogical tools will be generally defined as any instrument or technique that assists in the valid transmission of knowledge. Within the same discussion, Bernstein stated how "curriculum defines what counts as valid knowledge" (Sadovnik 1991:51). These definitions will be useful as a guide throughout this study when discussing curriculum (i.e. what is taught) or pedagogy (i.e. how it is taught).
} 
and mastering knowledge or skills. ${ }^{2}$ Faculty members' understandings/views will be understood as being largely influenced by their sociology department, ${ }^{3}$ graduate school experience, and interactions with colleagues. Sociology departments will be viewed as operating under institutional influences. ${ }^{4}$

Sociology faculty members learn what types of pedagogical tools may be viewed as legitimate by fellow academics, as well as what types of factors are important for student learning, through interacting with others within their respective departments and institutions. It is possible that departmental and institutional views can change over time. ${ }^{5}$ What may be viewed as a legitimate pedagogical tool at one time may not be at another. Similarly, what may be viewed as important to learning may also change over time. Currently, student engagement is believed to be of great importance for positive learning outcomes, but do sociology faculty members share this view? And, how do sociology faculty members today view the use of music in higher education sociology courses?

In my early college career, I found myself in an introductory sociology course with an unenthusiastic professor who spoke in a monotone voice and seemed to make an hour-long class double in time. If it had not been for my overall goal of attending law

\footnotetext{
2 Newmann (1992:12)

${ }^{3}$ Valimaa (1998) discussed how the cultural approach of higher education research consists of two traditions; 1) disciplinary-based cultures (i.e. where focus is given to the individual academic to reconstruct disciplinary cultures - more micro to macro, unit of analysis is disciplines),

4 and 2) institutionally based cultures (i.e. structured by higher education institutions - more macro to micro, unit of analysis is institutions). To fully understand academic behavior it is important to take both into account.

${ }^{5}$ Schatski (2002) discussed how practice is intrinsically social and the product of continuous interactions with others, and is constantly evolving.
} 
school, ${ }^{6}$ I would have probably changed my major. Thankfully, there were sociology faculty members in my undergraduate department who were able to successfully engage students with charisma, interesting life experiences, and critical thinking inquisitionsthis I believe helped immensely as I progressed toward attaining an undergraduate degree.

As I continue to advance my education and prepare for a career in teaching sociology to adult learners, I find myself pondering what can be done to create a more engaging learning environment in higher education sociology courses. Since I have a strong background in music (performing, producing, and as a disc jockey), I am especially interested in the incorporation of music into the classroom as a way to encourage and positively engage students with sociological content.

As stated by Zyngier (2004:2), "student engagement has been identified as an important precursor to student learning." Previous studies have suggested that student engagement positively influences educational achievement, academic outcomes, and may help protect students from dropping out of school (Fredericks et al. 2004).

Fredericks et al. (2004) discussed three types of student engagement found in recent literature; 1) behavioral engagement_-doing work and following rules, 2) emotional engagement — student levels of interest, values, and emotions, and 3) cognitive engagement - student motivation, effort, and strategy. Most relevant to this study is the emotional engagement experienced by students. I will first examine how a lack of emotional engagement (e.g. boredom) may impact student learning before moving on to

\footnotetext{
${ }^{6}$ In order to be a pre-law student at the University of Montana, one had to be enrolled in the sociology program with an emphasis in criminology.
} 
discuss how higher education sociology students may be effectively engaged in higher education sociology courses through using music as a pedagogical tool.

The need to promote emotional engagement among students appears to be a major issue within higher education. The Higher Education Research Institute's (HERI) annual surveys of college students continue to document high levels of academic disengagement. Recently, 36.4 percent of college students reported frequently feeling bored in class (Higher Education Research Institute 2011). This is up from a low of 25 percent in 1985 (Higher Education Research Institute 1999). Pekrun et al. (2002:97) discussed how boredom implies both physiological and cognitive deactivation, "thus leading to reduced attention and a more shallow, superficial processing of information." The researchers further discussed how negative deactivating emotions such as boredom, or hopelessness, may generally be detrimental because they erode motivation, or cognitive engagement, and direct attention away from the task such as learning course content. This erosion of motivation, inattention toward content, and cognitive deactivation associated with boredom can negatively affect student learning. With the recently reported high levels of emotional disengagement experienced by college students, and the potentially negative repercussions it has on learning, what can be done?

Analytically reflecting on ideas, questions, and discussions from a 2007 American Sociological Association teaching workshop of nearly forty sociologists, Macheski et al. (2008:42) discussed how "there is a need to create an environment that encourages students to be active and engaged participants in their own learning." The authors suggested sociology faculty members can encourage students to be active and engaged 
participants through "building a community of learners." That is, through developing an active role for the student, creating a common language of discourse, and building a supportive emotional environment. Maier (2013:2) found that "music can function as a classroom community-building activity as well as an educationally significant experience" in higher education English courses. This study seeks to contribute to a better understanding of how music as a pedagogical choice ${ }^{7}$ may be used by higher education sociology faculty members to keep students actively engaged, and assist in effectively 'building a community of learners' in higher education sociology courses.

On a physiological level, music has been shown to simultaneously tap into and stimulate both hemispheres of the $\operatorname{brain}^{8}$ (Berk 2008), link into image networks, cause synapses to fire more frequently (Zull 2008), enhance learning, stimulate lingual processes, and reduce students' levels of stress and anxiety (Keskin 2011). Through the way it effectively engages the brain, it appears that music can be used as a way to effectively engage college students.

In higher education sociology courses, faculty members have used music to illustrate important sociological concepts (Walczak and Reuter 1994), to foster group discussion and question assumptions about the students themselves and others (Martinez 1994), to promote active learning (Ahlkvist 1999), to aid in teaching students how to apply concepts and theories (Ahlkvist 2001, Elterman 1983, Hinds-Aldrich 2012,

\footnotetext{
${ }^{7}$ This study will choose to focus solely on pedagogy and how music as a pedagogical tool is viewed in relation to student engagement and learning by higher education sociology faculty members; while acknowledging that both curriculum and pedagogy are highly important in higher education sociology courses.

${ }^{8}$ That is, the logical and analytical left hemisphere processes rhythm and lyrics, while the non-verbal and creative right hemisphere processes the melody, sounds, and harmonic relationships of music.
} 
Martinez 1993, Walczak and Reuter 1994, Schneider 2011), to break student civil inattention and signal the beginning of class (Albers and Bach 2003, Dean 2012, and Palmer 2011), to illustrate societal inequalities (Bach and Albers 2010, Munkres 2010), to demonstrate contrasting viewpoints on a particular issue (Boggs 1993) or between different generations (Walczak et al. 1989), and as a way to humanize the professor while creating a shared meaning between students and faculty members (Albers and Bach 2003). Self-reported data suggested that music can be used to help engage sociology students, while increasing interest and aiding in the comprehension of course material (Ahlkvist 1999, 2001, Albers and Bach 2003, Elterman 1983, Hinds-Aldrich 2012, Martinez 1998, Reuter 1993, Walczak and Reuter 1994). While song lyrics have been generally incorporated into the discussion when faculty members have used music in their courses, lyrics have also been used by themselves to teach sociological content in higher education sociology courses; such as in teaching Marx's conflict theory (Walczak 1993), or content analysis methodology (Bach and Albers 2010, Shea 1993).

Although numerous studies suggested that music can be used as an effective pedagogical tool in higher education sociology courses, some also suggested that it is not very common (Hinds-Aldrich 2012, Martinez 1995, and Reuter 1993). As one student commented on the use of music in his higher education sociology course, he "really enjoyed this way of teaching - it is different from the way a majority of teachers teach" (Reuter 1993:81). Similar to Berk (2008), Cooper and Condon (2004:2) suggested using music is an uncommon practice in other disciplines in higher education as well, stating; "the irony is that so few educators utilize recorded resources," although "rock lyrics are 
“ear candy" to most students, and offer relief from bland traditional textbooks. If student engagement is viewed as important to student learning in higher education, and music has been shown to increase student engagement in higher education sociology courses, then why is music not used more often as a pedagogical tool? That is, what are the potential structural barriers that may inhibit faculty members' choices to use music as a pedagogical tool in their higher education sociology courses?

Structural barriers can potentially affect faculty members' decisions to use music as a pedagogical tool in higher education sociology courses, and in other disciplines as well. Structural barriers will be generally defined as institutional obstacles that prevent certain forms of pedagogy and/or pedagogical tools from being deployed. Potential structural barriers that may affect faculty members' choices to use music as a pedagogical tool include, but are not limited to; negative views by colleagues (Martinez 1993, and Walczak et al.1989), greater institutional/departmental focus on research than pedagogy (Becher 1989, and DeCesare 2003), and trouble with securing the proper audio equipment (Walczak et al. 1989). As Walczak, Alger, and Reuter (1989:5) warned; "Because popular music is not respected in many circles, including (especially) academia, you may face resistance and skepticism from your colleagues when you try to use music as a legitimate teaching technique." This type of academic skepticism has been found to exist in other sociology departments (Martinez 1993), as well as in other higher education disciplines (Bloom 1987, Cooper 1982, Keskin 2011, Pichaske 1999, and Terhune 1997).

To begin to answer the previous question of how music might be used in higher 
education sociology courses to assist in promoting student engagement, and in "building a community of learners," it is important to first learn more about faculty members' understandings of student engagement and learning. In addition, it is crucial to better understand how both music-using and non-music using sociology faculty members view the use of music as a pedagogical tool. Currently, little is known about how the use of music as a pedagogical tool is viewed by higher education sociology faculty members. Is the use of music viewed as a legitimate or illegitimate pedagogical tool among higher education sociology faculty members today? Do sociology faculty members feel their departments or educational institutions encourage or discourage certain types of pedagogical tools? And, do sociology faculty members view student engagement as an important precursor to student learning? To help address these types of inquiries, three research questions were developed.

\section{Research Questions}

This study seeks to answer three research questions:

$\boldsymbol{R Q 1 . ~ H o w ~ d o ~ h i g h e r ~ e d u c a t i o n ~ s o c i o l o g y ~ f a c u l t y ~ m e m b e r s ~ u n d e r s t a n d ~ t h e ~ r e l a t i o n s h i p ~}$ among pedagogical tools, student engagement, and student learning?

$\boldsymbol{R Q 2 . ~ H o w ~ d o ~ h i g h e r ~ e d u c a t i o n ~ s o c i o l o g y ~ f a c u l t y ~ m e m b e r s ~ u n d e r s t a n d ~ t h e ~ u s e ~ o f ~ m u s i c ~}$ as a pedagogical tool?

RQ3. How do higher education sociology faculty members understand structural barriers that might impact choices to use music as a pedagogical tool? 


\section{Summary}

In this chapter, the central issues underlying the research topic were introduced. That is, the importance of student engagement for student learning, music as a potentially beneficial pedagogical tool in higher education, and potential structural barriers that may impact the use of music as a pedagogical tool in higher education sociology courses. The basic research questions were presented as well. The following chapter consists of a review of relevant literature. 


\section{Chapter Two: Review of Literature}

This comparative interview study will explore higher education sociology faculty members' understandings of the use of music as a pedagogical tool, and of its relation to student engagement in higher education sociology classrooms. A review of relevant literature was completed to gain an understanding of how different sociology researchers view the use of music as a pedagogical tool. The literature review also explored previous studies' findings regarding how faculty members understood the relationship between pedagogical tools, student engagement, and student learning as it relates to the active learning approach. The literature review chapter is divided into three main sections: 1) active learning and other pedagogical tools used by higher education faculty members to promote student engagement, 2) the potential benefits, costs, and structural barriers that sociology faculty members have associated with using music as a pedagogical tool, and 3) the theoretical framework for this study. A brief summary discussing the relevance of each section in answering this study's research questions will be provided at the end of the chapter. 


\section{Active Learning and Other Pedagogical Tools Used to Promote Student Engagement}

\section{Active Learning}

Relevant articles related to the active learning approach were analyzed to provide a better understanding of how sociology faculty members may actively engage their students with the content, while promoting student learning in their courses.

In his meta-analysis of the approach, Prince (2004) defined active learning as any instructional method that engages students in the learning process. This definition refers to activities that are introduced into the classroom, while acknowledging the core elements of the active learning approach to consist of student activity and engagement in the learning process. The active learning approach promotes student engagement through capturing students' attention, which was found by Wankat (2002) to be roughly fifteen minutes during lecture. ${ }^{9}$ Courses that used interactive-engagement methods were shown to significantly increase student performance ${ }^{10}$ (Hake 1998), and brief activities have been shown to help students remember more content when introduced into lecture ${ }^{11}$ (Hartley and Davies 1978). In the end, Prince (204:226) found that "introducing activity into lectures can significantly improve recall of information while extensive evidence supports the benefits of student engagement" to increase student learning.

\footnotetext{
${ }^{9}$ As discussed by Zull (2002) this may potentially be due to habituation, where synapses in the brain fire less frequently if people hear the same sound over and over. Interest picks up when a new sound is introduced (e.g. another person talking, or a song being played).

${ }^{10}$ Hake found that test scores measuring conceptual understanding to be nearly twice as high in classes promoting student engagement compared to traditional courses.

${ }^{11}$ Hartley and Davies found the number of students paying attention drops dramatically during lectures resulting in a loss of retention in the lecture material (i.e. tests administered immediately after the lecture showed students remembered 70 percent of the information presented in the first ten minutes as opposed to 20 percent of the information presented in the last ten minutes).
} 
D'Silva (2010) expanded the discussion of the active learning approach to include other benefits as well, such as a students being able to connect the content to their existing knowledge. She defined the active learning approach as "models of instruction that focus the responsibility of learning on students by allowing [them] to engage in learning that promotes higher-order thinking” (p.81). In her analyses of the approach, D'Silva (2010:77) found; "students not only become engaged with the ideas being communicated among themselves, and between the students and the instructor, but also process the information acquired, integrate the newly acquired information with their prior knowledge, retain the integrated information over time, and subsequently transfer their learning to new situations.” D'Silva (2010) found that, through being actively engaged, students are able to process, connect with, retain and apply course content.

Bean (2011) defined active learners as those students who can apply course concepts to new problems, learn how to gather and analyze data, prepare hypotheses, consider alternative views, and formulate arguments through critical thinking tasks ${ }^{12}$ designed by the course instructor. One of the many strategies Bean discussed for designing critical thinking tasks involves linking new concepts back to a "structure of known material.” As past cognitive research has shown (Zull 2002, Bransford, Brown, and Cocking 2000, Norman 1980), "the more that unfamiliar material can be linked to the familiar ground of personal experience and already existing knowledge, the easier it is to learn" (Bean 2011:151). In his research, Bean (2011) illuminated how involving adults in the learning process (e.g. through engaging and critical thinking activities) and

\footnotetext{
${ }^{12}$ Critical thinking tasks included problems, questions, or issues for students to ponder or discuss.
} 
connecting unfamiliar content to students' existing knowledge is important when using the active learning approach. This latter point is highly relevant when discussing the following study.

While active learning appears to be considered highly beneficial for college student learning, a recent study suggested otherwise. Andrews et al. (2011) found the active learning approach to be widely accepted by higher education biology faculty members within their study, ${ }^{13}$ yet found no positive correlation between active learning activities and student learning. They explained this outcome by suggesting most college biology instructors lacked a full and rich understanding of the active learning approach. That is, most instructors are implementing only a portion of the active learning approach - activities to break up lecture - without necessarily linking the information with what students already know. The authors suggested that further training and understanding of the active learning approach by instructors is needed for the approach to be broadly successful in higher education institutions, thereby ensuring that all facets of the active learning approach are incorporated into classrooms to enhance student learning outcomes. Fascinatingly, the researchers found that student interest in the course was positively correlated with student learning. Although activities related to the active learning approach were not found by themselves to increase student learning, student engagement (interest) and active learning strategies were supported as effective teaching methods for promoting student learning.

\footnotetext{
${ }^{13}$ With $61 \%$ of instructors reporting using active learning activities at least once per class meeting.
} 
The findings from this section will be related to the use of music as a pedagogical tool in the next section. First, I will briefly discuss pedagogical tools that may promote or inhibit student engagement and active learning in higher education sociology courses.

Other Pedagogical Tools That May Promote Student Engagement and Active Learning

Bean (2011) described specific pedagogical tools that can promote student engagement and active learning in the higher education classroom. These engaging pedagogical tools mentioned by Bean included reflective writing, argumentative writing (where opposing views are considered), student as teacher, small group discussions, role playing, summaries of readings, case studies, and simulations. Some of these pedagogical tools will be covered in more detail in the remainder of this section.

\section{Small Group Work}

Prince (2004:229) suggested that small group work is highly beneficial for student engagement and learning, stating; "the best available evidence suggests that faculty should structure their courses to promote collaborative and cooperative environments," such as promoting small group work. Bean (2011:186) discussed how lecture by nature places students in a passive role, but when combined with small group work it can "help students learn both the conceptual and procedural knowledge and the discipline's ways of conducting inquiry and making arguments.” Other researchers found that small group activities involving purposefully designed and sequenced tasks produced higher levels of thinking than lecture (Bligh 2000, Hillocks 1986, and Hillocks, Kahn, and Johnnessen 
1983), whole class discussions, and nondirective group work (Hillocks 1986, and Hillocks, Kahn, and Johnnessen 1983). Bean (2011:185), although never directly addressing the use of music in higher education courses, described how during small group exercises he often finds his "view of a novel or a poem has been reshaped by the arguments of students."

\section{Simulation Games}

Dorn (1989) found simulation games ${ }^{14}$ can generate high levels of interest, empathy, motivation, improve interpersonal skills, and improve students' attitudes toward self, environment, and classroom learning. Dorn described how students are provided the analytical thinking needed to process the information gained from the experience during the post-game discussion. In this way, discussion of the games can help students connect the lessons learned to their own experience. It appears simulation games can promote student engagement through increasing student levels of interest and motivation in the course.

\section{Films and Television Shows}

Incorporating feature films into presentations is another helpful pedagogical tool sociology faculty members used to engage students. Groce (1992:80) discussed how feature films can be used to show the relevance of sociology to students' lives by bringing "a touch of familiarity to a seemingly foreign way of looking at and thinking

\footnotetext{
14 Simulation games are an activity where students role play an external reality through realistic characters and scenarios in the classroom.
} 
about the world." Groce (1992:82) described how the film One Trick Pony (1980) "has never failed to engross and engage students."

Incorporating episodes of television shows into class presentations is another pedagogical tool sociology faculty members have used to engage students in their own learning. Scanlan and Feinberg (2000) discussed how students become actively engaged in the learning process and class discussions because many of them are already familiar with a popular show like "The Simpsons" and watch it as a form of entertainment. The researchers noted that The Simpsons television show complements teaching philosophies that stress applying the sociological imagination to the observation of everyday life. ${ }^{15}$ By the end of the course, students commented how they could no longer watch the show recreationally due to their view of it being "contaminated" by its sociology. These studies illustrated that faculty members can use both film and television shows as pedagogical tools to engage students and provide a familiar connection to sociological content.

\section{Photographs and Music}

Hraba et al. (1980) described that music, when combined with visual images, can be helpful in engaging students and in bringing a historical period to life. These authors believed photographs and music could serve to provide the subjective view of social change to students, as opposed to faculty members' traditional approach of just presenting the objective facts and figures. Hraba et al. (1980) noted that music must be

\footnotetext{
15 This they state appears to be a common theme of the use of popular culture in sociology courses, including the use of music as a pedagogical tool.
} 
authentic in order to communicate a feeling of social change. They also provided a list of critical factors to take into account when selecting appropriate music for class presentations. Specific factors included; taking into account the tempo, clarity, and recording quality of the music, as well as the authenticity, substance, and salience of the lyrics. The authors mainly recommended the use of folk music, but also alluded to using classical music and music from the time period being discussed. They found that using a combination of music and slides as a pedagogical tool complemented the course materials on the Great Depression, promoted student receptivity and took students "beyond their own biographies." Hraba et al. (1980:130) noted that students inquired "why this sort of thing is not done in other courses, since it makes the class so real." In this study, the researchers found combining photographs and music as a pedagogical tool helped students engage with historical presentations, and move beyond their own biographies, while still allowing students to connect with the content in a 'subjective' manner.

So far I have focused on research about pedagogical tools that may help actively engage students in sociology courses. Now I will examine studies that discussed other pedagogical tools that might potentially hinder student engagement and active learning in higher education sociology courses.

Other Pedagogical Tools That May Inhibit Student Engagement and Active Learning Textbooks

Although textbooks are regularly used as a pedagogical tool, Shacht (1990) viewed the use of statistics textbooks as a barrier to student learning. He contended that 
the textbooks are largely written to engage the interests of professional sociologists and not students. Therefore the books have little, if any, pedagogical value for students. Shacht (1990) described how he stopped using a textbook in his statistics courses due to the books' lack of success in promoting student engagement with statistics, which he stated is generally perceived as a difficult subject by students. Shacht (1990) declared that sociology faculty members should select pedagogical tools that address the aforementioned problems and "reject those who do not."

\section{PowerPoint}

Hill et al. (2012) discussed the pros and cons of using PowerPoint slide presentations as a pedagogical tool in sociology courses. In this study, faculty members used PowerPoint primarily for providing clarity for students and to improve their own teaching performances. ${ }^{16}$ A third of the faculty participants reported that using PowerPoint was helpful for decreasing their own performance anxiety. The researchers discussed three potential dilemmas with using PowerPoint in the higher education sociology classroom. First, PowerPoint presentations tend to oversimplify course content. Second, relying on PowerPoint presentations often results in passive and disengaged students. Third, an over-reliance on PowerPoint presentations may create tension for faculty members between pragmatic career concerns and personal pedagogical philosophies (Hill et al. 2012). Several faculty members also noted that "institutional pressure to receive positive student evaluations of teaching lay at the heart of their

\footnotetext{
${ }^{16}$ For example, by helping faculty members stay on track during lecture.
} 
compromise" to use PowerPoint over their preferred pedagogical techniques (Hill et al. 2012:253). This study suggests that perceived institutional pressures and student demands may influence faculty members' choices with regard to selecting and incorporating pedagogical tools into their sociology courses.

I now turn toward discussing in what ways higher education sociology faculty members have used music as a pedagogical tool to promote active learning and student engagement in their courses.

\section{Using Music as a Pedagogical Tool: Potential Benefits, Costs, and Barriers}

Numerous studies suggested that music is an effective pedagogical tool for engaging students in higher education sociology courses (Ahlkvist 1999/2001, Boggs 1993, Bowman 1993, Dean 2012, Elterman 1983, Hinds-Aldrich 2012, Martinez 1993/1994/1998, Palmer 2011, Shae 1993, Walczak 1993, and Walczak and Reuter 1994). Some of these studies specifically mentioned how music can be "engaging" for students, or serve as a "catalyst for learning," while others discussed other aspects associated with the active learning approach.

Music has been used in sociology courses in ways consistent with an active learning approach, including promoting student engagement and connecting content to students' existing knowledge. This section is divided into sub-categories covering the potential benefits, costs, and barriers associated with the use of music as a pedagogical 
tool. How faculty members have chosen to overcome the potential costs and barriers will also be discussed.

\section{Potential Benefits}

Several benefits of using music as a pedagogical tool in higher education sociology courses have been identified, including: increasing student engagement and interest in sociology, encouraging discussion, promoting active learning, illustrating concepts and theories, enhancing the sociological imagination of students, providing a connection to students' individual lives, and other benefits to be discussed later. I will begin by discussing how music may promote student engagement.

Multiple studies found that music can effectively increase student interest in sociology (Ahlkvist 1999, Elterman 1983, Hinds-Aldrich 2012), make the material more enjoyable (Ahlkvist 2001, Walczak and Reuter 1994) and facilitate discussion and student participation in higher education sociology courses (Ahlkvist 2001, Elterman 1983). As Walczak and Reuter (1994:267) stated; "In general, [students] found the use of music in the classroom to be a refreshing, relevant, entertaining, thought-provoking, and effective way to learn sociology." Other studies found that music may increase levels of engagement through discussion in higher education sociology courses (Martinez 1998, Palmer 2011). As Palmer (2011) stated, "music can pull your students into a discussion, get them to consider controversial issues from new perspectives, and set a tone for a great class.” Martinez (1998:210) suggested creating a list of questions to help start a discussion for any particular song. She noted; "the discussion of these questions leads to 
further questions that generate more and more relevant discussion linking the lyrical content to the community, the society, and history."

Several studies found that music is helpful for illustrating and clarifying sociological concepts (Ahlkvist 1999, Martinez 1994, Reuter 1993, Walczak et al. 1994, Walczak and Reuter 1993) and increasing student understanding of the material (Martinez 1994, Walczak and Reuter 1993) by helping to make the concepts more “accessible" (Bach and Albers 2010, Burns and Martinez 1993). Further, even when students did not particularly like the music being played it was still believed to be an effective pedagogical tool for illustrating sociological concepts (Reuter 1993, Walczak and Reuter 1994). This could be potentially due to music providing a tangible link for students to unfamiliar and abstract theories (Ahlkvist 2001), by giving them something familiar (i.e. songs and lyrics in general) to connect with.

Multiple studies found support for the usefulness of music in developing the sociological imagination of students (Boggs 1993, Dean 2012, Elterman 1983, Martinez 1998, Walczak and Reuter 1993) while helping to connect concepts to the individual lives of students and their existing knowledge base (Bach and Albers 2010, Boggs 1993, Elterman 1983, Martinez 1995, Munkres 2010, Walczak 1993, Walczak et al. 1989). Burns and Martinez (1993:117) argued that the use of examples from popular culture in the social theory classroom can stimulate the "sociological imagination ${ }^{17}$ of the student by underscoring the relevance of social theory in everyday life." Discussing the use of music as a pedagogical tool, Burns and Martinez (1993:126-7) stated; "the incorporation

\footnotetext{
${ }^{17}$ Coined by C. Wright Mills (1959:3) the term stresses the importance of connecting individual events with the broader social processes, as he stated; "...neither the life of the individual nor the history of society can be understood without understanding both."
} 
of such examples [from popular music] into the classroom offers the opportunity to add interest and relevance to the course content, especially for that part of the student body for whom such theory is most foreign."

Researchers have identified other benefits as well for using music as a pedagogical tool in higher education sociology courses. Specific benefits included how the use of music can; enhance student levels of critical thinking (Walczak 1993), be less overwhelming than using films (Hinds-Aldrich 2012), imply faculty members care about students and their learning (Albers and Bach 2003), be an important avenue of communication between faculty members and students (Burns and Martinez 1993), and enhance learning through helping students retain course content (Ahlkvist 2001).

Ahlkvist (2001) found that the use of progressive rock music enhances learning when explicitly integrated into the classroom. Ahlkvist's (2001:479) findings suggested that, "the technique eases students' introduction to social theory and helps them retain a basic understanding of the difficult material."

While the literature is fairly extensive regarding the potential benefits of using music in higher education courses, less focus has been given to the potential costs and structural barriers associated with using music as a pedagogical tool.

\section{Potential Costs}

Previous researchers have identified several potential costs associated with the use of music as a pedagogical tool in higher education sociology courses. Specific costs included; the technique is time consuming, students may question the type of music being 
played, faculty members may not like the music, students may be offended and shutdown, song lyrics may be limiting, or students may not take the use of music as a pedagogical tool seriously.

Albers and Bach (2003:241) described how "it took considerable amount of musical research to select the songs to be played for each class period.” Martinez (1995) echoed this sentiment when discussing how linking music to course content may be challenging. Ahlkvist (2001:481) disagreed and suggested that faculty members can easily integrate progressive rock music into their courses using the songs from his study, because intimate knowledge of the genre is not needed and preparation time is "minimal." Several researchers suggested including students in the song selection process, which adds songs to faculty members' sound banks and helps to keep the music relevant for students (Albers and Bach 2003) while being a great way to get students to take an "active role" in the class (Palmer 2011).

One potential cost had to do with the type of music faculty members used in their courses. Walczak and Reuter (1994:267) discussed that "most of the negative comments [by students] concern the type of music played." Ahlkvist (2000) noted that students viewed older progressive rock music as confusing and largely irrelevant for understanding their current social environment. Hinds-Aldrich (2012) discussed how using music not preferred by students may lead them to engage less deeply then using familiar genres, but found a potential way to limit this by giving instructions and setting the stage prior to listening to a song. Walczak and Reuter (1994) suggested using a greater variety of music by including songs that do not solely criticize the social order, 
involving students more deeply by requiring them to select music, and having students think more critically about the music itself and the cultural context or political agenda that the music may be representing. However, Walczak et al. (1989) warned that involving students in the song selection process could lead to faculty members having to listen to music that may not be to their taste.

Martinez (1994:263-4) discussed how "music that strongly questioned institutions and stereotypes often elicited the most emotional responses." While most student emotional responses were positive and insightful, some students voiced their feelings regarding the music selections by stating Martinez (1998:211) "had an ethnic chip on her shoulder," or characterized her as "a "bitch" [while] stating that racial and ethnic groups complain about problems that the nation had already solved." Ahlkvist (1999:128) specifically chose to incorporate heavy metal into his classroom due to the prevailing negative stereotypes about its fans and performers that provoke strong (usually negative) feelings, which as Ahlkvist explained, guarantees "high student interest and involvement from the outset."

When using potentially controversial music, Martinez (1998) suggested that faculty members explain how the music specifically relates to the lecture material prior to it being played, and to allow students to leave if they feel uncomfortable. Palmer (2011) also discussed setting the stage by telling his students "to be prepared for artistic expressions that may surprise, shock, or potentially offend them." He also described how he invests in censored versions of songs, or if a song feels "too risky" he chooses not to play it. 
Elterman (1983:536) warned to "avoid selecting songs in which the lyrics cannot be clearly understood...or in which significant parts of the song's lyrics may be confusing or ambiguous." This ambiguity and confusion could lead to decreased learning outcomes. To help alleviate the potential ambiguity of song lyrics, several studies suggested to provide a hard copy of the song lyrics to students (Albers and Bach 2003/2010, Boggs 1993, Elterman 1983, Martinez 1993/1998), or at least display them when discussing a song (Albers and Bach 2003/2010). Boggs (1993:19) stressed to "ALWAYS have the words printed and copied for distribution to the students. The point you are trying to make will be lost in an inferior recording...the lyric may be buried under 110 trombones and not readily apparent in one or two playings."

The last potential cost that will be discussed in this section involves students not taking the use of music as a pedagogical tool seriously. As Ahlkvist (1999:140) found "the most significant challenge is persuading some students to take the analysis of heavy metal seriously...rather than as an amusing diversion.” Initially students may not take the technique seriously, but as Ahlkvist (1999:140) later described; "once we get through the first application of Marx and Durkheim to heavy metal, the majority of students understand the logic of cultural analysis.” Elterman (1983:536) also discussed how students may not take the use of songs as seriously as they should, stating; "after all, the information is not coming from a textbook or a professor's lecture but from songs generally listened to for entertainment."

Overall, researchers perceived the potential benefits to outweigh the costs, especially when music is properly used as a pedagogical tool by directly relating the 
music to the course content, discussing why a particular song was played, and displaying the song's lyrics for student analysis and discussion.

\section{Potential Structural Barriers}

For the purpose of this study, structural barriers are defined as departmental and institutional obstacles that prevent certain forms of pedagogy and/or pedagogical tools from being deployed. The lack of training, support, or resources can all be viewed as potential structural barriers. A lack of training, support, or resources may also prevent higher education sociology faculty members from understanding the available pedagogical tools to use in their courses. These types of barriers can influence faculty members' views of pedagogical tools and how they relate to student learning. Previous research has identified several structural barriers for the use of music as a pedagogical tool. Specific barriers included; professional socialization and the lack of value placed on teaching, negative views of colleagues, music viewed as an uncommon practice, as well as copyright laws and a potential lack of access to audio equipment.

\section{Professional Socialization and Lack of Value Placed on Teaching}

One potential structural barrier for the use of any pedagogical tool that is discussed in several non-music related studies involves the nature of professional socialization and the lack of value placed on teaching in higher education. Several studies suggested that higher education sociology faculty members are socialized during graduate school to focus primarily on research; at the same time faculty receive little 
instruction in pedagogy (Atkinson 2001, DeCesare 2003, and Rau and Baker 1989). The heavy emphasis on research in graduate student professional socialization is believed to negatively impact faculty members' knowledge of pedagogy. As Rau and Baker $(1989: 163,172)$ discussed, "the problem is that faculty are neither trained, motivated, nor rewarded for providing high quality instruction," which leads pedagogy to "never receive the time, effort, and creativity it requires." Although Atkinson (2001:1226) believed teaching is beginning to increase in importance, she voiced the same concerns as Rau and Baker over a decade later, stating; "under the current system of valuing only traditional research, if we spend our time working on our teaching skills, we are in effect punished because we have less time to devote to traditional research." Similarly to Atkinson, and another decade later, Chalmers $(2011: 35,32)$ found while "in a relatively short time, in university terms, there has been consistent and substantial progress" made regarding the acceptance, appreciation and recognition of the scholarship of teaching, "the evidence suggests that on measures of perception, promotion, tenure and salary, teaching continues to be relegated to a lower status with promotion, tenure and higher salary directed to those who do disciplinary research and publish." It is through research and the publication of findings where academics gain prestige and credibility, and excellence in teaching counts little towards recognition from established colleagues (Becher 1989). Rau and Baker (1989) also discussed how a dualist perspective may affect the pedagogical choices of higher education faculty members. The authors described how dualists expect faculty members to give the correct facts and answers to problems, without discussing the possibility of more than one correct answer or several legitimate 
perspectives regarding the same problem. Rau and Baker (1989:171) believed this dualism bears an affinity toward time-saving teaching practices, leading to a mechanical routinization of teaching and the socializing of students into "a culture of intellectual sloth.”

Professional socialization and the lack of value placed on teaching in higher education may potentially affect sociology faculty members' views of the use of music as a pedagogical tool in a few ways. First, if sociology faculty members are socialized to focus primarily on research then implementing non-traditional pedagogical tools such as music may be viewed as not important when compared to pursuing a research agenda. Second, due to limited training in pedagogy, sociology faculty members may not fully understand how the use of music can enhance student learning in the classroom and may not view it as a legitimate pedagogical tool. Third, the mechanical routinization of teaching may lead some faculty members to choose efficient, yet non-engaging, pedagogical tools for their courses.

\section{Negative Views of Colleagues}

Another potential structural barrier found within the literature is the degree to which specific pedagogical tools are viewed as legitimate by faculty members within a particular department. Martinez (1993:62) discussed her colleagues' reactions to her use of music as a pedagogical tool, stating; "most were supportive, however, I also got the distinct impression that this was considered a little "eccentric."” Martinez (1993:72) also described how "colleagues have tentatively been suggesting that this might not be so bad 
after all" and how using music as a pedagogical tool led her to become a finalist for the College Superior Teaching Award. Although Martinez was able to overcome the negative views of her colleagues, ${ }^{18}$ her experience illuminates how other sociology faculty members may view the use of music as an illegitimate pedagogical tool, which could potentially hinder its use.

Walczak, Alger, and Reuter (1989:2) also discussed how music may be viewed negatively by colleagues as "just another gimmick aimed at pandering to student whims." The researchers warned how "popular music is not respected in many circles, including (especially) academia, you may face resistance and skepticism from your colleagues when you try to use music as a legitimate teaching technique" (Walczak et al. 1989:5). This suggests that sociology faculty members may not view the use of music as a legitimate pedagogical tool, and this skepticism and resistance may also affect how other faculty members view the use of music.

\section{Music Viewed as Uncommon Practice}

Another potential barrier for the use of music as a pedagogical tool is that it is viewed as not common practice in higher education classrooms. Martinez (1995:415) speculated; "although I suspect that students often sense the implications of song lyrics at some level, they seldom have the opportunity to discuss them in an academic setting." Berk (2008:63) commented how "music is virtually an untapped resource for teaching the Net Generation." Although it receives minimal attention in the literature, it appears from

\footnotetext{
18 That is, her colleagues' negative views of music as a pedagogical tool as "eccentric," and in the statement "it might not be so bad after all" - alluding that the use of music was viewed as illegitimate by her colleagues at some point.
} 
the previous statements that music either is not used very often or is not perceived to be used very often in higher education sociology courses. This can serve as a potential barrier for the incorporation of music as a pedagogical tool in sociology courses. If music is not used very often by higher education sociology faculty members then faculty members may not realize its potential educational benefits, or may potentially view it as an illegitimate pedagogical tool used by only a few rogue faculty members. Interestingly, regardless of how frequently music is used, if it is perceived as not being a common practice the results will be the same.

\section{Copyright Laws and a Potential Lack of Access to Audio Equipment}

Other potential structural barriers were mentioned during discussions with faculty members at Portland State University. During these conversations a lack of knowledge of copyright laws and a potential lack of access to audio equipment were cited as potential barriers. The professor who voiced concern about copyright laws was unclear about the legality of publicly playing copyrighted music in her/his classrooms, and whether or not he/she could legally distribute the songs on a compact disc to students. Similar to Walczak et al. (1989), another professor mentioned how it used to be difficult to find proper audio equipment in the classrooms to playback music. ${ }^{19}$ Both of these potential barriers illustrate how the lack of knowledge regarding copyright laws, and/or access to audio-visual equipment, can serve as potential structural barriers for faculty members seeking to incorporate music into their courses. These types of barriers may

\footnotetext{
${ }^{19}$ Martinez (1993:62) was able to overcome the access to AV equipment barrier by buying her own "boom box" from the local JC Penney department store.
} 
affect faculty members' choices to use music as a pedagogical tool. That is, if faculty members are not informed of copyright laws, or do not have access to proper equipment, they may be unlikely to choose to use music as a pedagogical tool in their courses.

The first two sections of this literature review chapter examined the findings from studies on; 1) active learning and other pedagogical tools used by higher education faculty members to promote student engagement, and, 2) the potential benefits, costs, and structural barriers that sociology faculty members have associated with using music as a pedagogical tool. The final section of the chapter will present the theoretical framework for this study.

\section{Theoretical Framework}

In his book Mind, Self \& Society, Mead (1934) discussed how shared meaning develops through the process of communication and social interaction. When an individual makes a gesture it has a specific idea behind it. When that gesture is accurately interpreted by another person - that is when it arouses the same idea in the other person - a shared significant symbol is created and a unifying connection is made between the two parties. It is through these types of connections with other individuals where meaning is created, as Mead (1934:75-6) described:

"A gesture by one organism, the resultant of a social act in which the gesture is an early phase, and the response of another organism to the gesture...constitutes the matrix within which meaning rises. The gesture 
stands for a certain resultant of the social act, a resultant to which there is a definite response on the part of the individuals involved therein; so that meaning is given or stated in the response...Meaning is implicit—if not always explicit—in the relationship among the various phases of the social act to which it refers..."

The meaning of social acts are not fixed, they are continually emerging and shaped through our interactions with others and our ability to create meaning through shared symbols. These shared symbols are social objects (ideas or things) created and legitimated through interaction with others, where "meaning is given or stated in the response." As Mead (1934:249) stated; "when we call out [a] response in others, we can take the attitude of the other and adjust our own conduct to it." The meaning of a social object (e.g. the use of music as a pedagogical tool) is dependent on the response given by others. For example, if other faculty members view music as an illegitimate pedagogical tool then a faculty member may take the attitude of the others and adjust their own views to it. That is, he/she may also begin viewing the use of music as an illegitimate or "unacademic" pedagogical tool. Mead described this as taking the role of the generalized other; that is, taking the role of a social group which one identifies with.

Through Mead's work, one can see how legitimacy is gained through social interactions with others. This is applicable to sociology faculty members as well. That is, the sociology faculty members one associates with may influence how he/she views the use of music as a pedagogical tool. Also, one may have different social groups they identify with that may lead them to view a social object differently in different social 
settings. That is, what may be viewed as a legitimate pedagogical tool in one social setting may not be so in another. A technique that is viewed as a legitimate pedagogical tool by students in a classroom may not be viewed as such by other faculty members in a department. Higher education sociology faculty members learn what is viewed as acceptable for different social settings through social interactions with others.

\section{Summary}

This chapter began by discussing student engagement and student learning, as well as the active learning approach. It was found in the literature that student engagement, as well as connecting content to students' existing knowledge, is associated with increased student learning. Previous research found that some pedagogical tools increased student engagement and learning in sociology courses, such as music, simulation games, science fiction novels, television shows, films, and photographs and music, while others such as statistics textbooks and PowerPoint potentially hindered student engagement in sociology courses. The potential benefits, ${ }^{20} \operatorname{costs}^{21}$ and barriers ${ }^{22}$ associated with the use of music as a pedagogical tool were also identified in relevant literature. The understandings gained from the review of relevant literature will be

\footnotetext{
${ }^{20}$ Potential benefits included: increasing student engagement and interest in courses, encouraging discussion, promoting active learning, illustrating concepts and theories, and enhancing students' sociological imagination.

${ }^{21}$ Potential costs included: how the technique is time consuming, students may question the type of music being played, faculty may not like the music, students may be offended and shutdown, discussions of particular music genres may lead to stereotyping by students, or students may not take music as a pedagogical tool seriously.

22 Potential barriers included: a lack of value placed on teaching, negative views of colleagues, music being viewed as not common practice, a lack of knowledge of copyright laws, as well as a lack of access to audio equipment.
} 
insightful when exploring the research questions for this study. This literature review will also be useful when discussing whether or not music may be used in higher education sociology courses to build a community of learners. Potential gaps identified in the reviewed literature include; 1) a lack of research regarding how sociology faculty members perceive the use of music as a pedagogical tool, such as how users and nonusers understand the technique as it relates to benefits, costs, and barriers, and 2) a lack of data regarding the actual usage of music as a pedagogical tool in higher education sociology courses.

Finally, Mead's (1934) theory was discussed to help understand how higher education sociology faculty members may create and associate meaning to the use of music as a pedagogical tool. This study will contribute to the discussion of whether or not music is perceived as a legitimate pedagogical tool by different groups of sociology faculty members in different departments by exploring faculty members' understandings of how others may influence the meaning they associate with certain pedagogical tools. More specifically, what meaning do users and non-users of the technique assign to the use of music as a pedagogical tool in higher education sociology courses? Does faculty members' shared meaning of music as a pedagogical tool affect faculty members' choices to use music in their courses? Interviewing matched-pairs of sociology faculty members in similar departments should lead to a more complete understanding of how sociology faculty members understand the perceived benefits, costs, and barriers associated with the use of music as a pedagogical tool. The next chapter presents a detailed description of how this study will be conducted. 
This chapter presents an overall description of the project design and methodology used in this study. Included in this chapter are descriptions of the setting, participants, recruitment procedures, operational definitions and interview guides used. Data analysis will also be discussed prior to proceeding to the following chapter covering findings and results.

\section{Justification for using a Qualitative Approach}

This study focused on the individual lived experiences of two groups of sociology faculty members (i.e. those who used music as a pedagogical tool on a consistent basis ${ }^{23}$ in their courses - "DO," and those sociology faculty members who did not—-"DN") to illuminate the unexplored phenomenon of how the use of music in higher education sociology courses is viewed by sociology faculty members in general. A qualitative approach is an appropriate choice for this study since it can yield a deeper understanding of the processes and complexities of lesser-known phenomena, while assisting in illuminating relevant variables that are yet to be defined for such phenomena (Marshall and Rossman 2011).

\footnotetext{
${ }^{23}$ Using music as a pedagogical tool on a consistent basis is defined as using music across the span of an entire sociology course, or across multiple courses.
} 
Another strength of a qualitative approach is that it allows participants to describe the meaning of their own lived experiences (Marshall and Rossman 2011). As stated by Marshall and Rossman (2011:91); "human actions cannot be understood unless the meaning that humans assign to them is understood." Since not much is known about how sociology faculty members understand the use of music as a pedagogical tool (e.g. the potential benefits, costs, and structural barriers related with the technique), and what using music in higher education sociology courses means to sociology faculty members (e.g. how they generally view the use of music and what meaning they assign to the use of music as a pedagogical tool), a qualitative methodological approach is the most appropriate choice for this study as it allows participants to describe the meaning of their own understandings and experiences. These collective individual meanings and understandings were then analyzed for themes to illuminate how the sociology faculty members in this study generally viewed the use of music as a pedagogical tool.

\section{Project Design}

This study used a comparative interview design. As stated by Ragin and Amoroso (2011:55); "the goal of exploring diversity is best served by the comparative approach." A comparative design was an appropriate choice as this study focused on the similarities and differences in how two groups of sociology faculty (i.e. those who used music consistently as a pedagogical tool, and those who did not) understand the use of music in the sociology classroom; for example, whether they see music as potentially related to 
student engagement, and what structural barriers (if any) do they perceive as potentially inhibiting the use of music in higher education sociology courses. Through using a comparative design, new insights can be obtained and potential explanations may develop for the existence of certain phenomena (Thomas 2009); in this case, the use of music as a pedagogical tool.

In-depth interviews were also used to explore faculty members' ideas about what pedagogical tools they preferred to use in their courses, whether or not they viewed student engagement as important for student learning, what tools they believed promoted student engagement, and whether they believed music might be used as an effective tool for promoting student engagement in higher education sociology courses.

\section{Research Questions}

This study attempts to address three research questions:

\section{$R Q 1$. How do higher education sociology faculty members understand the} relationship among pedagogical tools, student engagement, and student learning?

In what ways are the understandings of the relationship among pedagogical tools, student engagement, and student learning of faculty members who use music on a consistent basis as a pedagogical tool similar to and/or different from those of faculty members who do not use music consistently as a pedagogical tool? 


\section{RQ2. How do higher education sociology faculty members understand the use of}

music as a pedagogical tool? In what ways are the understandings of the use of music as a pedagogical tool among faculty members who use music consistently as a pedagogical tool similar to and/or different from those of faculty members who do not use music consistently as a pedagogical tool?

\section{RQ3. How do higher education sociology faculty members understand structural} barriers that might impact choices to use music as a pedagogical tool? In what ways are the understandings of the structural barriers that might impact choices to use music as a pedagogical tool among faculty members who use music consistently as a pedagogical tool similar to and/or different from those of faculty members who do not use music consistently as a pedagogical tool?

\section{Setting}

This research was conducted at eight different colleges and universities across the U.S. and Canada ${ }^{24}$ where selected participants taught sociology courses using music as a pedagogical tool. In two cases, a satellite campus of the same institution was used for comparison. Most of the selected colleges and universities identified as being public institutions, with only two identifying as being private-i.e. one sectarian and one nonsectarian. Most of the schools were smaller in size, meaning the student population was

\footnotetext{
${ }^{24}$ Seven schools were located in the U.S. That is, two in the West, two in the Northeast, two in the South, and one in the Midwest. And one school was located in Western Canada.
} 
under ten-thousand students. ${ }^{25}$ The institutions varied regarding whether they considered themselves a research university (three), a liberal arts university (two), an applied arts college (one), a four-year college (one), or a two-year college (one). The size of the sociology departments also varied by institution, ranging from zero to twenty-nine tenure-track faculty members.

\section{Participants}

Overall eighteen higher education sociology faculty members completed interviews for this study. ${ }^{26}$ One matched pair was excluded from the study, ${ }^{27}$ leaving sixteen cases to be analyzed. Most participants for this study were tenure-track higher education sociology faculty members. In some cases non-tenured or part-time faculty members were selected due to a limited number of tenure-track appointments, ${ }^{28}$ or, due to a lack of a tenure-track at one of the institutions. Since the purpose of this study is to explore higher education sociology faculty members' understandings of the use of music as a pedagogical tool, of its relation to student engagement in higher education sociology classrooms, and of the potential structural barriers that may exist, it makes sense that participants were sociology faculty members. When possible, tenure-track faculty members were chosen as participants due to being potentially more knowledgeable about

\footnotetext{
25 Two schools were just above ten-thousand students, while one served over thirty-thousand students. These population numbers include both undergraduate and graduate students.

${ }^{26}$ Four pilot interviews were also completed locally - with two sociology faculty members for each group.

27 Due to one professor no longer using music as a pedagogical tool in his courses, and it being unclear whether the other participant did or did not use music consistently in his courses.

28 "In the United States, 69\% of academics now work off the tenure track" (Levin and Shaker 2011)
} 
their department and institution than part-time faculty members (Levin and Shaker 2011, Thirolf 2012). For more participant characteristics, please see Tables 1 and $2 .^{29}$

Table 1: Participant Demographic Characteristics $(\mathrm{N}=16)$

\begin{tabular}{|c|c|c|c|c|c|c|}
\hline & $\begin{array}{l}\text { Decade } \\
\text { Born }\end{array}$ & Sex & $\begin{array}{c}\text { Race/ } \\
\text { Ethnicity }\end{array}$ & $\begin{array}{l}\text { Position } \\
\text { Held }\end{array}$ & $\begin{array}{l}\text { \# Years } \\
\text { Teaching } \\
\text { at Inst. }\end{array}$ & $\begin{array}{c}\text { Courses Currently } \\
\text { Teaching }\end{array}$ \\
\hline $\mathrm{DO}$ & $\begin{array}{l}1-1950 \\
1-1960 \\
4-1970 \\
1-1980\end{array}$ & $\begin{array}{l}4-\mathrm{F} \\
3-\mathrm{M}\end{array}$ & $\begin{array}{l}6 \text { - White } \\
1 \text { - Latina }\end{array}$ & $\begin{array}{c}\begin{array}{c}\text { 5- Tenure- } \\
\text { track }\end{array} \\
1-\text { Full-time } \\
1-\text { Full-time } \\
\text { temp. }\end{array}$ & $\begin{array}{l}1-22 \\
1-14 \\
1-13 \\
1-5 \\
1-4 \\
1-3 \\
1-<1\end{array}$ & $\begin{array}{l}6 \text { - Intro. } \\
2 \text { - Race and Ethnicity } \\
2-\text { Methods } \\
2-\text { Deviance } \\
2 \text { - Social Problems } \\
1 \text { - Theory } \\
1 \text { - Social Psych. }\end{array}$ \\
\hline $\mathrm{DN}$ & $\begin{array}{l}1-1940 \\
2-1950 \\
1-1960 \\
4-1970 \\
1-1980\end{array}$ & $\begin{array}{l}7-\mathrm{F} \\
2-\mathrm{M}\end{array}$ & $\begin{array}{l}7 \text { - White } \\
1 \text { - Latina } \\
1 \text { - Black }\end{array}$ & $\begin{array}{c}6-\text { Tenure- } \\
\text { track } \\
1-\text { Full-time } \\
1-\text { Adjunct } \\
\text { Lecturer } \\
1-\text { Part-time }\end{array}$ & $\begin{array}{l}2-12 \\
1-9 \\
2-5 \\
2-2 \\
2->1\end{array}$ & $\begin{array}{l}8 \text { - Intro. } \\
1 \text { - Race and Ethnicity } \\
3 \text { - Methods } \\
2 \text { - Deviance } \\
2 \text { - Social Problems } \\
1 \text { - Theory } \\
1 \text { - Social Psych. } \\
2 \text { - Women's Issues } \\
1 \text { - Gender }\end{array}$ \\
\hline
\end{tabular}

As shown in Table 1, participants for both groups were primarily white, female and held tenure-track positions. Sociology faculty members who used music consistently as a pedagogical tool tended to be younger, and have more teaching experience at their current institutions than those who did not use music consistently as a pedagogical tool. Regarding the types of courses that each participant currently taught, most participants from both groups were likely to teach an introductory sociology course. Those sociology faculty members who did use music consistently as a pedagogical tool were more likely

${ }^{29} \mathrm{DO}=$ higher education sociology faculty members who do use music consistently as a pedagogical tool, $\mathrm{DN}=$ higher education sociology faculty members who do not. $\mathrm{Y}=\mathrm{Yes}, \mathrm{N}=$ No. 
to teach courses on race and ethnicity (two) than those who did not (one). Those sociology faculty who did not use music consistently as a pedagogical tool were more likely to teach courses on women's issues (two - Introduction to Women's Studies and Women in Crime), Gender (one) and Research Methods (three) than those who did use music consistently as a pedagogical tool (i.e. two faculty taught Research Methods). Both groups appeared to be equally similar regarding teaching Deviance, Social Problems, Theory, and Social Psychology. Other noteworthy courses taught by faculty who did not use music consistently as a pedagogical tool, and not by faculty who did, included Medical Sociology, Mental Illness, Biology and Society, and Statistics. Noteworthy courses taught by faculty who did use music consistently as a pedagogical tool, and not by faculty who did not, include Inequality, Homelessness, Sociology of Aging, Sexuality, and The Sociology of Food and Obesity. 
Table 2: Participant Graduate School Characteristics ( $\mathrm{N}=16)$

\begin{tabular}{|l|l|l|l|l|}
\hline & \multicolumn{1}{|c|}{$\begin{array}{c}\text { Grad School } \\
\text { Location }\end{array}$} & $\begin{array}{c}\text { Grad School } \\
\text { Type }\end{array}$ & $\begin{array}{c}\text { Grad School } \\
\text { Size }^{31}\end{array}$ & $\begin{array}{c}\text { Completed } \\
\text { Teaching } \\
\text { Course? }\end{array}$ \\
\hline DO & $\begin{array}{l}3-\text { Midwest } \\
3-\text { Northeast } \\
1-\text { West }\end{array}$ & $\begin{array}{c}7-\text { Public } \\
\text { Research } \\
\text { University }\end{array}$ & $\begin{array}{l}5-\text { Medium } \\
2-\text { Large }\end{array}$ & $\begin{array}{l}7-\mathrm{Y} \\
0-\mathrm{N}\end{array}$ \\
\hline DN & $2-$ Midwest & $7-$ Public & $1-$ Small & $4-\mathrm{Y}$ \\
& $2-$ Northeast & Research & $3-$ Medium & $5-\mathrm{N}$ \\
& $1-$ South & University & $5-$ Large & \\
& $2-$ West & $1-$ Private & & \\
& $1-$ France & Research & & \\
& $1-$ Canada & University & & \\
& & S- Private & & \\
& & Sectarian & & \\
& & Liberal Arts & & \\
& & University & & \\
& & & & \\
\end{tabular}

Table 2 illustrates the similarities and differences of participants' graduate school experiences. Most participants from both groups attended medium to large public research universities in the United States. Faculty who did not use music consistently as a pedagogical tool were more likely to attend a private university, both of which were located in the U.S., and less likely to have completed a teaching course than those faculty who did use music consistently as a pedagogical tool.

\footnotetext{
${ }^{30}$ While five faculty members attended separate institutions for their Master's and Doctorate degrees, only the institutions where participants earned (or will earn) their Doctorate degrees were taken into account for this table due to participants most likely spending more time at the institutions where they were earning their Doctorate degrees. Locations are categorized mainly by U.S. region as specified by the U.S. Census Bureau.

${ }^{31}$ Small Institutions are categorized by total enrollment being $<10,000$ students (including undergraduate and graduate students), Medium Institutions range between 10,000 and 30,000 students, and Large Institutions include $>30,000$ students.
} 


\section{Participant Identification and Selection}

Participants were selected using stratified purposeful and snowball sampling techniques (Marshall and Rossman 2011). Participants who used music as a pedagogical tool in past or current courses were initially identified through various literature, websites, teaching resources, or by word of mouth. ${ }^{32}$ These potential participants were then contacted via email to complete a sixty to ninety minute interview. If multiple email attempts failed to produce a response, participants were then contacted via telephone. Once a response was given and an interview date/time was confirmed, the participant was then emailed a copy of the informed consent document to peruse prior to interviewing. During the interview, participants were asked if they had read the informed consent document and willingly gave their consent to be interviewed for the study. Near the end of the interview, participants were asked if they would be willing to recommend someone from their department who is "demographically similar" and "who is unlikely to use music in their courses." Upon completion of the interview, participants were awarded a ten dollar iTunes gift card as an incentive for their participation.

Participants who did not use music consistently as a pedagogical tool were generally identified by their colleagues who did use music during the completed interview. In two cases, those referring their colleagues chose to do so anonymously. In these situations, a portion of the initial email was changed from "you have been referred by...to participate" to "you have been referred by a colleague to participate", or, "you

\footnotetext{
32 One participant was identified by a former colleague as having used music before and was contacted then assigned an interview slot. During the interview the participant disclosed he had not used music before, and then completed the interview using the second interview guide for participants who did not use music consistently in their courses. In this case (matched pair \#2), recruitment occurred in the reverse order.
} 
have been selected to participate." When the referred faculty members declined to be interviewed, the researcher would then contact the referring participant for other suggestions. When no suggestions were given, as was found in three cases, the researcher contacted appropriate faculty to complete an interview using "you have been selected to participate in a study exploring pedagogical tools and student engagement," while attempting to select faculty members who did not use music as a pedagogical tool in their courses. Participants who did not use music as a pedagogical tool were selected to match the participants who did use music in their sociology courses as best as possible, to ensure that both groups are more or less comparable to one another with regards to potential structural barriers within a similar institution. In all, sixteen faculty members' completed interviews were analyzed in this study; that is, seven who used music consistently as a pedagogical tool in their higher education sociology courses, and nine who did not. ${ }^{33}$

\section{Data Collection}

Interviews were conducted between August $1^{\text {st }}$ and October $31^{\text {st }}$ of 2012, using semi-structured open-ended interviews. The semi-structured format allowed questions to be asked in a logical sequence, while still providing space for the exploration of emergent ideas raised by participants. This method of data collection balanced the need to collect

\footnotetext{
33 Originally both groups consisted of eight participants, but during analyses it was discovered that one DO group member did not use music on a consistent basis (i.e. showing music videos on occasion in a popular culture course, and only using lyrics — not songs — in an introductory course to analyze and illustrate deviance).
} 
readily comparable data with the need to allow for gaining rich data through thick descriptions and fully exploring unanticipated and relevant topics.

Confidential in-depth interviews were conducted in one of three ways; 1) in person, 2) via Skype (a free online video conferencing service), or 3) by telephone. Interviews were conducted in person when feasible, while most interviews were conducted via Skype or by telephone. ${ }^{34}$ Skype was chosen as the preferred method, as both parties can read one another's non-verbal cues via the video screen as dialogue is exchanged. Due to being the most impersonal data collection technique, telephone interviews were used as a last resort or when participants explicitly stated that would work best for them. Whether in person, via Skype, or by telephone, every effort was made to ensure that participants were located in a comfortable and familiar environment of their choice.

Interview questions were asked in an authentic and casual manner, while humble inquiry, ${ }^{35}$ probes, and prompts were used as needed to elicit further information.

\section{Instrument Design}

Two separate interview guides ${ }^{36}$ were constructed for the two groups of faculty members for this study (i.e. higher education sociology faculty members who used music consistently as a pedagogical tool, and those who did not). Both interview guides were

\footnotetext{
34 Eight DN group faculty members chose to be interviewed via telephone, with one DN participant interviewing via Skype. Three DO group faculty members chose to be interviewed via Skype, three chose to be interviewed via telephone, and one DO group participant interviewed in person.

${ }^{35}$ Greene (2005)

${ }^{36}$ Copies of both instruments are included in appendix A and B.
} 
significantly informed by the review of the literature and carefully driven by the research questions for this study. A planned and necessary revision of each interview guide occurred after data were collected from four pilot interviews- with the most notable outcome being a shift toward a greater focus toward pedagogical tools, and less toward pedagogical approaches. ${ }^{37}$ Each interview guide was structured in a similar manner; with both the introductory questions, background/education section, and the closing questions being identical in wording and sequence. The identical sections of the two interview guides are described solely within the discussion of interview guide number one, the guide used with those higher education sociology faculty members who did use music consistently as a pedagogical tool in their courses.

During all interviews operational definitions were given when requested by the participant, or when further clarity was needed such as when the participant was unsure of the question, took a long pause, or appeared perplexed. These definitions were extremely useful, especially in giving concrete examples for what qualifies as a pedagogical tool. Usually participants were very receptive, and the given examples helped spark ideas and insights in participant responses. The operational definitions used are as follows ${ }^{38}$ :

\footnotetext{
${ }^{37}$ When asked about pedagogical approaches, the majority of participants would begin naming pedagogical tools in lieu of actual approaches. Overall, in both pilot and other interviews, faculty members were more knowledgeable about pedagogical tools and less knowledgeable about approaches. This also held true for faculty who completed courses on "effective approaches for teaching at the collegiate level," where minimal focus was usually given toward pedagogy and pedagogical approaches, and more focus was given toward the practicalities of teaching a college sociology course.

38 Drawing from Bernstein's discussion on how "pedagogy defines what counts as valid transmission of knowledge," and how "curriculum defines what counts as valid knowledge" (Sadovnik 1991, p. 51), along with the review of applicable literature, these definitions and examples were constructed.
} 
Operational Definitions

- $\quad$ The Use of Music $=$ songs and lyrics.

- $\quad$ Pedagogy $=$ the valid transmission of knowledge.

- Pedagogical Approach = how faculty members choose to transmit knowledge (e.g. Socratic or dialectic inquiry, Critical — critique/deconstruction of knowledge, Constructivist — constructing knowledge with students, Experiential—relating knowledge to experience, etc.).

- $\quad$ Pedagogical Tool $=$ any instrument or technique that assists in the valid transmission of knowledge (e.g. Syllabi, PowerPoint Presentations, Handouts, Film, Music, Literature, Poetry, etc.).

- $\quad$ Structural Barrier $=$ institutional obstacles that prevent certain forms of pedagogy and/or pedagogical tools from being deployed (e.g. lack of training, a/v resources, knowledge of copyright laws, colleague support, etc.).

- Curriculum $=$ what counts as the valid transmission of knowledge.

During each interview, a copy of the informed consent document and the above operational definitions were set aside for review as needed. 


\section{Interview Guide I}

Introductory Questions: The first section was designed to ensure that participants qualified for the study, and read the informed consent document. Participants were asked their current position, how long they have been teaching at their institution, and which sociology courses they currently taught.

Background/Education: The second section focused on participants' graduate school courses. The first question was intentionally left broad to allow participants to recall what was most memorable about their time spent in graduate school courses. The probes and following questions then "funneled" down and became more narrow to explore what types of pedagogical approaches and tools were engaging for participants during their graduate coursework. The rationale was to explore what types of pedagogical tools were used, and which were engaging for participants, during their time of professional socialization in graduate school. Some faculty members chose to include some background and discussion regarding their undergraduate sociology courses: this allowed for further inquiry into participants' educational background in sociology and added to the discussion.

Teaching Experience and Music as a Pedagogical Tool: The focus in the third section shifted to the current experiences of participants in their role as a faculty member. Participants were asked to discuss their views on the relationship between student engagement and learning, and to support their positions using examples from personal experience (which led to further insight regarding the ways sociology faculty members choose to engage students). Questions regarding the types of pedagogical tools 
participants chose to use in their classrooms were also included, followed by a detailed discussion regarding the use of music in their courses (e.g. costs, benefits, and how they feel music impacts learning). The rationale behind such discussions was to fully explore the use of music (e.g. looking for costs and benefits that do not appear in the literature), while also including other pedagogical tools used by participants that they most likely found effective in teaching sociology. Participants were asked for "guesstimations" and speculations regarding the percentage of higher education sociology faculty members who use music in their courses ${ }^{39}$ (both for their department and in the U.S.). In addition, a follow-up probe asked participants if they can speculate as to why only the identified percentage of faculty members (usually below 20\%) used music as a pedagogical tool in their courses. The rationale behind these types of questions were to explore whether or not the use of music is common in higher education sociology courses (a discussion not commonly found in the reviewed literature), and to have faculty members preemptively discuss any potential barriers that they feel may hinder the use of music as a pedagogical tool. The final question explored whether or not participants will continue to use music in their courses, giving room for discussion regarding how they might use it differently in the future.

Structural Barriers and Other Pedagogical Tools: In the fourth section, participants were asked to discuss the range of pedagogical tools used by other faculty members in their respective departments. A follow-up question asked participants how they felt the use of music was viewed by their colleagues. Participants also discussed if

\footnotetext{
${ }^{39}$ No data was found in the reviewed literature that identified the frequency of sociology faculty members using music as a pedagogical tool.
} 
some pedagogical tools were encouraged or discouraged by their department or institution. Here, the intention was to illuminate whether or not faculty members, departments, or higher education institutions discourage the use of music in higher education sociology classrooms, and thus serve as a potential barrier for the use of music by faculty members. Included in this section were also questions regarding structural (i.e. institutional and departmental) barriers that participants may have encountered when utilizing music in their courses, and how they chose to overcome these barriers.

Closing Questions: In the fifth section, demographic questions were included to gather data about participants' age, race/ethnicity, and other variables for potential future analyses. Demographic characteristics may be useful in the transferability of the understandings gained from this study to similar studies with similar questions of practice, or, to similar faculty located in similar departments and institutions (Marshall and Rossman 2011). Also included in this closing section, respondents were given time to ask questions of the interviewer and recommend other higher education sociology faculty members for the study.

\section{Interview Guide II}

Introductory Questions: Same as above. Background/Education: Same as above.

Teaching Experience and Pedagogical Tools: The third section of this interview guide was very similar to the one used in Interview Guide I, save that focus shifted toward participants' self-chosen "preferred pedagogical tool." Participants were asked to 
discuss the costs, benefits, and possible impacts regarding learning and student engagement associated with using this pedagogical tool. The final question of the section asked if respondents are likely to continue using their preferred pedagogical tool in the future. The rationale behind this section is to identify the perceived benefits, costs, and barriers associated with participants' preferred pedagogical tools and compare them to the perceived benefits, costs, and barriers associated with the use of music as a pedagogical tool.

Structural Barriers and Other Pedagogical Tools: The fourth section was also very similar to the same section in Interview Guide I. Here participants were asked about the pedagogical tools preferred by other faculty members in their departments, as well as any possible structural barriers that might exist for their preferred pedagogical tools. This section was also designed to be used in a comparative fashion with responses from the same section in Interview Guide I (i.e. discussing how possible structural barriers may affect other pedagogical tools as well).

Music as a Pedagogical Tool: Section five focused on the use of music as a pedagogical tool in higher education sociology courses. Participants were asked if they knew other faculty members who use music, and whether they see it as an effective tool for promoting student engagement in sociology courses. They were also asked to discuss their views on the relationship between the use of music as a pedagogical tool and learning. Similar to Interview Guide I, participants were asked to "guesstimate" the percentage of other sociology faculty they felt used music as a pedagogical tool—and then speculate as to why "only" that percentage did so. The final question asked 
participants if they would be likely to use music in their courses in the future, and for further explanation. This final question is important for future discussions regarding whether or not music may be used as a pedagogical tool in higher education sociology courses to assist in actively engaging students and 'building a community of learners.'

Closing Questions: Same as the above.

\section{Analysis}

This comparative study employed incident-by-incident coding (Charmaz 2006) by identifying recurrent themes within the data, and patterns within those themes to help build overarching categories and an in-depth understanding of how the use of music as a pedagogical tool is viewed by higher education sociology faculty members. Preliminary categories were developed through analyzing relevant literature, and a preliminary codebook was established. As initial interviews were completed, general categories were identified through the open coding process described below (along with patterns within the general categories) and the codebook was updated as needed to fit the data. This was an on-going process until no new categories or patterns emerged.

During the analysis process, each confidentially transcribed interview ${ }^{40}$ was read at least two times from beginning to end, and codes were assigned to all incidents relevant to the research questions. When possible in vivo codes were assigned; that is, participants' direct language and special terms were used as codes to help preserve the

\footnotetext{
${ }^{40}$ All identifying data was removed (e.g. names, locations, etc.).
} 
meaning of their views (Charmaz 2006). Focused coding was then utilized for each individual interview to identify the most significant and/or frequent codes within that specific set of data. After being successfully coded, each interview was then compared with other interviews within the same group (e.g. higher education sociology faculty who used music in their courses) to identify themes and patterns for each group of faculty members. Once the themes and patterns were identified for both groups, codes were then collapsed into larger themed categories. During this process, and for the remainder of the data analysis, a modified version of the constant comparative method from Boeije (2002) was utilized. This method involved a 5-step comparison process: 1) comparison within a single interview (using incident-by-incident coding), 2) comparison between interviews within the same group (using axial coding), 3) comparison of interviews from different groups (using triangulation of data sources), 4) comparison within matched pairs (using selected themes from open coding), and 5) comparison between matched pairs (finding criteria to compare matched pairs).

After establishing separate themes and patterns for each group, the themes and patterns for the separate groups were then compared with one another to illuminate how those sociology faculty members who used music consistently as a pedagogical tool were similar and different from those sociology faculty members who did not use music consistently as a pedagogical tool. The similar themes found between the groups represent how sociology faculty members in general may view the use of music as a pedagogical tool in higher education sociology courses. Further analysis within matched pairs (i.e. between faculty members who used/did not use music consistently within the 
same institution) and between matched pairs (i.e. between matched pairs from different institutions) was also conducted to ensure all categories and patterns within the data emerged.

During data analysis, self-reflection of the researcher's personal experiences and the recognition of possible preconceptions were used to check internal biases. Analytic memorandums were used to track coding, clustering, and reflections. Through using analytic memos, reasoning behind choices were recorded (e.g. collapsing categories). This greatly assisted in the reflection process, and in maintaining a clearly organized path toward the completion of the study.

Also helpful during the analysis process was the use of investigator triangulation to check inter-coder reliability (Thomas 2009). Having another colleague or committee member occasionally code a completed interview, served as an external check to ensure coding categories were accurately defined. Throughout the process I frequently referred back to three texts for methodological guidance (i.e. Charmaz 2006, Ragin and Amoroso 2011, and Thomas 2009). Although these texts largely discussed international macro comparative designs, they also suggested that similar techniques can be used for more micro-level studies such as this one. For further assistance with this study, I also referenced Boeije (2002). 


\section{Summary}

This chapter described the data and methods used for this research study. The descriptions of the methods, data collection, and analysis process showed the rationale and decision making that went into the creation of the methodology for this study. In the next chapter, the specific results will be presented. 


\section{Chapter Four: Findings}

This chapter presents the findings of this study and is organized into three major sections. Each section will revisit a separate research question and present the key similarities and differences found between the two comparative groups (i.e. sociology faculty members who used music on a consistent basis_- "DO," and sociology faculty members who did not use music on a consistent basis in their courses_-"DN"). A figure will also be included at the beginning of each section to visually illustrate these key similarities and differences. After each research question has been revisited, a general summary of the findings will be provided. 


\section{RQ1. How do higher education sociology faculty members understand the relationship}

\section{among pedagogical tools, student engagement, and student learning?}
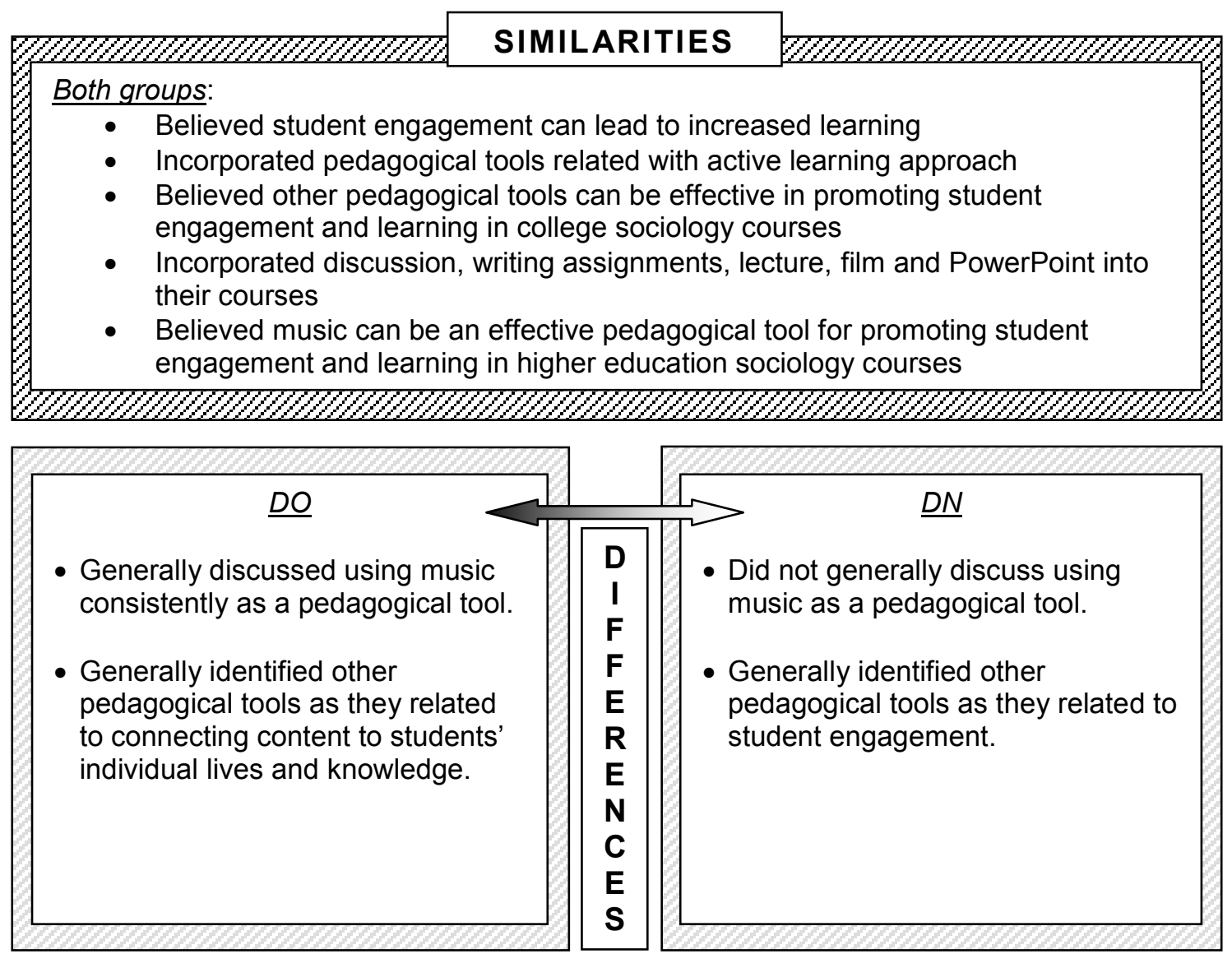

Figure 1: Similarities and Differences Between Faculty Groups: Pedagogical Tools, Student Engagement, and Student Learning.

\section{Key Similarities}

Overall, all faculty members from both groups believed student engagement can lead to increased student learning. One faculty member from each group expressed that student engagement has to be "genuine" or must "relate back to the course concepts" in order to be effective for student learning, but both believed student engagement could lead to increased student learning. 
Most faculty members incorporated at least two pedagogical tools related to the active learning approach (i.e. discussion and writing assignments) while also generally incorporating film, lecture, and PowerPoint into their courses. The pedagogical tools faculty viewed as relating to student engagement, and thus increasing student learning, were initially illuminated through the probe; "can you please give an example from your experience?" When answering this question, both groups of faculty members generally discussed non-music related examples ${ }^{41}$; illustrating how faculty generally viewed other pedagogical tools as being potentially effective in promoting student engagement and learning in higher education sociology courses. The majority of faculty members also viewed the use of music in a similar fashion.

Both groups of faculty members believed music can be an effective pedagogical tool for promoting student engagement and learning in college sociology courses. Almost all faculty members in both groups described how they believed music can promote student engagement and learning-even when some admitted that they had not previously thought about it. As two DN group faculty members noted:

"Now that I think about it, I think using that Woody Guthrie song as an example, you could actually augment it that way, promote engagement...I guess I never thought about it much before right now, but sure, thinking about some of those examples it could."

\footnotetext{
41 The majority of faculty members from the DO group chose to give non-music related examples (e.g. socio-biography, games, discussion, service learning, and video montage projects), while many faculty members from the DN group chose to give discussion related examples along with film, class response systems (e.g. clickers), visual images, and enthusiastic lecture.
} 
“Yes, I guess I kind of do think so. I don't think I ever thought about it until I peer reviewed his class, and then started thinking about my assignments, but yeah, I think it probably could.”

Other faculty acknowledged that they believed music can promote student engagement and learning, even though they did not view themselves as necessarily capable of integrating music into their own courses. As two other DN group members stated:

"I think it could have a positive impact on learning...I'm just not sure how to apply it in the classroom."

"I think it can be done in a very positive way. Unfortunately, I don't have the imagination to do it."

Other examples of how music may promote student engagement and learning will be discussed in a subsequent section that focuses on potential structural barriers for the use of music as a pedagogical tool in higher education sociology courses.

\section{Key Differences}

While many similarities existed between the two faculty groups regarding how they understood the relationship among pedagogical tools, student engagement, and student learning, differences were also be found.

The first key difference is how both groups of faculty members generally discussed the benefits of other pedagogical tools. DN group faculty members mentioned student engagement as it related to other pedagogical tools, more so than connecting 
content to the individual lives of students. ${ }^{42} \mathrm{DN}$ group members demonstrated this in two ways: First, through the pedagogical tools they chose to discuss and the explanations they provided when asked to give an example of student engagement increasing learning outcomes. And second, how they discussed the benefits of their preferred pedagogical tools-where the only general benefit found among DN group members' preferred pedagogical tools involved increasing student engagement.

On the other hand, DO group faculty members generally discussed other pedagogical tools as they related to connecting content to the individual lives of students such as by sparking students' sociological imagination. They demonstrated this by their choice of pedagogical tools, and the explanations they provided, when asked to give an example of how student engagement may lead to increased learning (e.g. sociobiography, video montage project, documentaries, and music). One DO group faculty discussed how an athlete engaged in her sociobiography ${ }^{43}$ assignment:

“He was totally engaged in the class...he didn't know how to write all the jargon that other students knew how to write, but he was able to capture the experiences of racism, of racism as a man of color, [and] as an athlete."

DO group faculty members also generally agreed that music may be used as a way to connect content to the individual lives of students and their existing knowledge base, as did DN faculty members as well.

\footnotetext{
42 Both of which are important aspects associated with the active learning approach.

43 This is a biography assignment that asks students to discuss how race, gender, and class have impacted their lives.
} 


\section{RQ2. How do higher education sociology faculty members understand the use of}

music as a pedagogical tool?
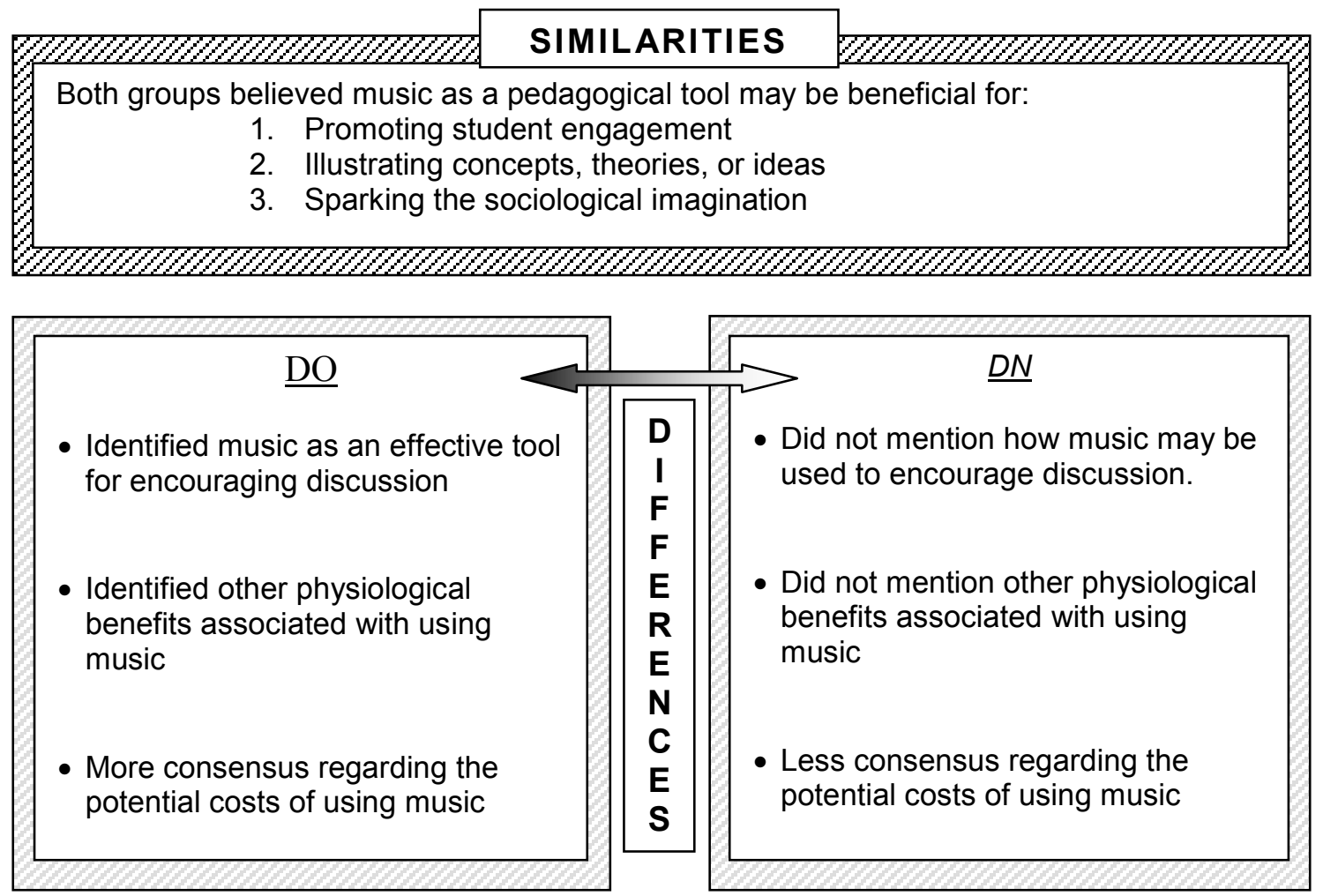

Figure 2: Similarities and Differences Between Faculty Groups: Benefits and Costs of Using Music as a Pedagogical Tool.

\section{Key Similarities}

Both groups of faculty believed music can potentially; 1) increase student engagement, 2) illustrate sociological concepts, theories, or ideas, and, 3) spark the sociological imagination. As discussed earlier in this chapter, DN group faculty members agreed that music has the potential to increase student engagement even when they lacked knowledge as to how it may do so. DO group faculty members generally provided 
more comprehensive examples regarding how music may increase student engagement in the sociology classroom. One DO group faculty member noted:

"It lends itself to every subject in sociology, it's actually the perfect tool to make the students at least listen...what I find when I play music more so than when I play movies is that they have a tendency to get off their phones. I find that really helpful to get them refocused."

This faculty member was able to get students to refocus on the course content by using music to engage students' listening skills. Most DO group faculty members discussed engaging students with the content through connection (i.e. sparking the sociological imagination), while others were a little more general in their comments regarding how music may potentially increase student engagement. Another DO group faculty member mentioned:

"I can't imagine thinking of music in any other way than a vehicle for engaging students and enhancing the learning environment, and then helping us achieve those [positive] learning outcomes."

Both groups of faculty members also generally discussed student engagement through using music as it related to other benefits as well, such as how music may be used to help illustrate sociological concepts.

Faculty members from both groups identified that music may be used to effectively illustrate sociological concepts, theories, or ideas. DO group members were more likely to discuss using music to illustrate sociological theories. As one DO group faculty member described: 
"They don't think about structural functionalism or conflict theory, but when they listen to music that is clearly conservative or very liberal, it makes it easier for them to understand...They don't know who Jello Biafra is, and after I play that I will play some American country music; God bless the USA for example, in order to illustrate the differences between structural functionalism and conflict theory, their different approaches for looking at the same thing, but through very different glasses."

DN group members were more likely to discuss using music to illustrate specific concepts, such as deviance:

"I put them in small groups and either they're all talking about the same set of lyrics, or like with the deviance thing I picked different songs that I thought represented deviance very differently and had them talk about those, different groups got different sets of lyrics, then they had to explain to the class what their lyrics were and read parts of the lyrics they found particularly [illustrative]; so what argument were they making about, did they think deviance was being presented as good or bad, and who did they think the audience was that was imagined listening to this song. Then they had to explain which lyrics in the song led them to this conclusion, just talking about deviance and sociology generally, and some of them would bring up themes like gender and sexism that I didn't necessarily ask them to." 
While both groups of faculty members viewed music as an effective pedagogical tool for illustrating particular theories or concepts, some also identified how it can help students discuss other unintended sociologically relevant topics or how it may be incorporated into the classroom in a variety of ways. One DO group faculty member explained:

"It's more than just the content of playing music. That's the same similar kind of thing to just giving the content, so you can use it in a bunch of different ways. You can criticize or critique lyrics for instance, so you can talk about rap music as a way to discuss something like the emergence of critical race theory and connect those things. You can use it to highlight or spotlight issues of censorship..."

Along with the ability to illustrate sociological concepts, theories, or ideas; both faculty groups agreed that music may be used as a pedagogical tool to help students "connect" with the course content.

As discussed in Chapter Two, the term sociological imagination stresses the importance of connecting individual events and broader social processes—-where students come "to see the relevance of social theory in their everyday life," Burns and Martinez (1993:118). In this manner, both groups of faculty believed in the potential of using music to spark the sociological imagination. One DO group faculty member stated:

"I'm not sure about much but I'm sure about needing to use lots of different tools to light their sociological imagination, turn it on. Music helps that cause...we're trying to get them to think sociologically, we're 
trying to get them to think in ways that involve structural explanation, we want them to think about institutions."

Some faculty members in the $\mathrm{DN}$ group also discussed how students may connect with the content through using music as a pedagogical tool. One DN group member observed:

"I think that students relate to music. I think it's something they feel kind of connected to even if it's not necessarily the music they're listening to that day...in my evaluation I commented on how much I feel like that was a strong connection. It was a connection to the topic, it was a connection to the instructor, and it was a culture class, so it was a connection to culture, and he [uses music] in all of his classes."

Here this faculty member illustrated how music may help students connect with sociological content, as well as with sociology faculty. One DO group participant discussed more specifically how students may connect with music on both personal and historical levels, stating;

"[Music] pulls students in, their everyday lives, it makes them relevant in the classroom rather than having these distinct separate things...music also is historical, so it connects to a certain place and a certain time and this is a real nice and easy demonstration of something like the sociological imagination, being able to question the social circumstances that give rise to the music, or the censorship of the music." 
While both groups of faculty reached a general consensus regarding some of the benefits associated with using music as a pedagogical tool, DO group members generally discussed additional benefits as well as a potential cost related with the technique.

Key Differences

There are two key differences regarding how each faculty group understands the use of music as a pedagogical tool, both involved knowledge of benefits and costs. First, DO group faculty members generally identified how the use of music may encourage discussion along with other physiological benefits. Second, DO group faculty members discussed the potential costs of using music by mentioning how faculty may "miss the mark" when using music as a pedagogical tool. DN group faculty members did not generally mention either of the additional benefits or the cost identified by the DO group.

The first additional benefit that DO group members identified is how music may encourage classroom discussion. DO group faculty members commonly related discussion with other identified benefits as well; such as, enhancing discussion and the sociological imagination through using music as a pedagogical tool. As one DO group faculty member discussed:

"It gets them to talk to each other and have conversations about why you like this music, and it goes beyond just oh, I like the beat. People talk about why they like certain beats and why they're drawn to certain types of music, and then we start talking about all the social forces that shape 
their individual preferences, and using our sociological imagination with them sitting around kind of chatting."

Other DO group participants described how music may help encourage discussion through relaxing students and faculty alike. As one DO group faculty member pointed out:

"Beyond it was just the cool thing to do? I feel like music has always been a part of my culture and my scene, and it alleviates anxiety both for me and I feel for my students. It kind of breaks the ice, leads into a nice discussion about the topic if we can introduce it with some form of music."

The second additional benefit DO group members mentioned involved the potential physiological benefits that music can provide. DO group faculty members discussed how the use of music as a pedagogical tool may help create an exciting, comfortable, and fun learning environment. One DO group participant noted:

"I think that it's a really effective pedagogical tool because as soon as you start to play music, students' faces light up, and in fact if you're going to do lecture all the time, which I do a lot, I mean it better be interesting, otherwise you're going to lose your students, and by the way, faculty really do lose students in a class, all they have to do is keep going on and on..." 
Another DO group faculty member discussed how music can create a relaxing environment, while also describing how some students may begin to question why it is not used by other faculty as well, stating:

"I play music at the beginning of every class. I ask them to tell me what their favorite songs are on the first day of class and I play them throughout the semester, this helps create a type of environment where they feel comfortable, like one they feel comfortable because they're hearing music in the class and they feel like it's relaxing, this is actually what they told me. They're like, 'Why can't every professor do this? It's so relaxing at the start of class'..."

Other potential physiological benefits DO group faculty members observed in their courses when using music included witnessing; students "perk up," a "collective spirit in the class," students having "more time to process" ideas while making learning "more exciting," and increasing levels of student interest while seeing music as something that "stays with them." As one DO group faculty member voiced:

"I think music helps with [learning sociology], and as I mentioned earlier, maybe provides for them a memorable classroom experience."

While DO group faculty members did spend a fair amount of time discussing the potential benefits of using music as a pedagogical tool, they also acknowledged that this is not without potential cost.

The last difference found between faculty groups involved the potential costs of using music in the classroom. DO group faculty members generally described how one 
may "miss the mark" when using music. This involved how students may potentially resent song selections (e.g. they may not be a fan, they may view it negatively, or view the music as outdated), become emotionally stressed (e.g. they may be offended by a song), choose music with negative stereotypes (e.g. when selecting music for a class project), or believe it is ok to use inappropriate language (e.g. if a song contains inappropriate lyrics). One DO group faculty member mentioned:

"Sometimes you misfire, same as with any other teaching strategy, sometimes you just miss the mark. It's happened to me many times, not just with music, there's been a million times where I just was like, man that was botched. It just didn't work. There was no execution, I missed the mark wildly, I was way off, I was unclear how I presented that idea. Ya know, It came off as nothing more than filler. It came off as just I'm some goofy guy playing a song. This is just a meaningless exercise. As with anything else, you can blow it."

The above faculty member described how the potential cost of using music may also be applied to other pedagogical tools as well. Additionally, DO group faculty members discussed how students may view the use of music negatively. Another DO group faculty member noted:

"Some people may think that it's stupid, you know 'what can I learn from Brad Paisley, or Bob Dylan,' or whatever. They may think it's a waste of time, they may want me to get right to what's going to be on the test or what's in the textbook, explain it to me, that sort of stuff. So, I think there 
can be a negative reaction to it and I think you risk, if you don't have good reasoning behind why you're using it, that students might react negatively to it, see it as either a waste of time or this 'guys crazy,' or something like that."

While they generally identified the potential cost of using music as a pedagogical tool, DO group faculty generally viewed the potential benefits to outweigh the potential costs. As one DO group faculty member stated:

"If it's a cost/benefit analysis, I see it as mostly a benefit. My experience in the last ten years that I've been teaching, it's always been overwhelmingly positive. Students have remarked how they have never thought of that before, and that's always something that makes me happy in terms of music."

Later in the discussion chapter I will explore how this cost may potentially be alleviated, but first I will discuss the potential structural barriers faculty members identified as being associated with the use of music as a pedagogical tool in higher education sociology courses. 
RQ3. How do higher education sociology faculty members understand structural barriers that might impact choices to use music as a pedagogical tool?
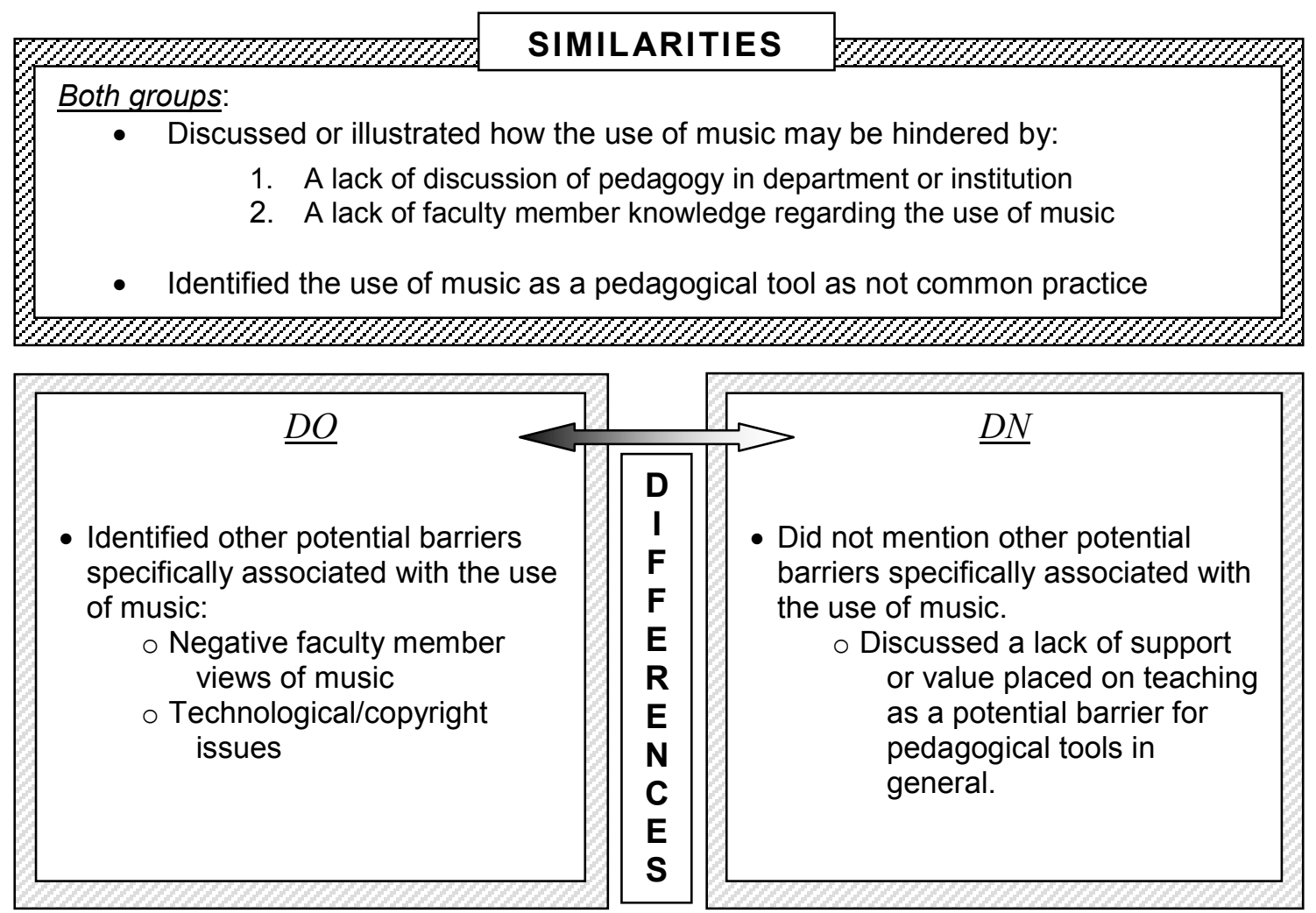

Figure 3: Similarities and Differences Between Faculty Groups: Potential Structural Barriers Associated With Using Music as a Pedagogical Tool.

\section{Key Similarities}

Faculty members from both groups discussed potential structural barriers that may be associated with the use of music as a pedagogical tool. Faculty members generally identified, or illustrated, that a lack of discussion of pedagogical tools both among colleagues and in completed teaching courses may serve as a potential barrier for incorporating music into one's sociology course. Both groups of faculty members also 
identified, or illustrated, that a lack of faculty knowledge regarding the use of music as a pedagogical tool may serve as a potential structural barrier. Further, faculty members also generally believed the use of music as a pedagogical tool to be not common practice in higher education sociology courses, which may also serve as a barrier.

Both groups of faculty generally believed, or illustrated, how there is a lack of discussion of pedagogical tools at the departmental or institutional level. This is substantiated through most faculty participants not being knowledgeable about what pedagogical tools their colleagues used ${ }^{44}$ or how they lacked knowledge regarding how their colleagues viewed their use of music or preferred pedagogical tool. When asked about what pedagogical tools were used by her colleagues, One DN faculty member stated:

"I don't really talk to my colleagues that much about what they do, so I don't think I would really be able to answer that question...I don't think I could speak for them. You probably know that the [University] is a research one university, it's excruciatingly focused on output of publications and getting grants, and so most faculty are really focused on those things, so the kind of casual conversations that come up among faculty tend to be more around that. There's not much discussion about teaching effectiveness and different pedagogical strategies."

\footnotetext{
${ }^{44}$ Besides generally discussing how they "don't know" what their colleagues are using in their courses, faculty members identified less pedagogical tools used by their colleagues (approximately half of what they cited as using in their own teaching experience). This may also be evidenced by only two faculty members from the DN group being knowledgeable of their colleagues' use of music as a pedagogical tool.
} 
This lack of discussion of pedagogical tools was also found in smaller departments as well (among both full-time and adjunct faculty). When asked about his colleagues' use of, and views regarding, music as a pedagogical tool, one DO group participant noted:

"There are two full-timers, myself and one other, and I don't know if she uses music. I don't think we've ever talked about that...we have a number of adjunct faculty, I'm not sure about their practices either. I'm not familiar...I really don't know. I wonder if some of them would take [music] seriously as a pedagogical tool?"

While a lack of discussion of pedagogical tools with colleagues was found in both groups of faculty, a lack of discussion of pedagogical tools was found in completed teaching courses as well. Faculty members who completed a course on effective teaching at the collegiate level generally cited a lack of discussion of pedagogical tools in said courses. When asked if the completed course covered any approaches to pedagogy, one DO group faculty explained:

"Not really, and that's probably one thing that I would have changed. I think there are a lot of different ways you could teach that course...there wasn't a lot of discussion about things that do or don't work in the classroom, no readings from Teaching Sociology. None of that really." If pedagogical tools were discussed, it was usually in a "traditional" sense (i.e. syllabus, lecture, exams) or in a very limited fashion. One DN group faculty member recalled only one tool where strengths or weaknesses were minimally discussed (i.e. after stating how the course did not cover any pedagogical tools or approaches): 
"We didn't really analyze which tools are most effective. I do remember talking briefly about PowerPoint and how students actually don't seem to do very well when you use PowerPoint in the classroom, but really just briefly talking about that. The class was more structured for us to figure out what kind of teacher we are already, instead of teaching us what is the best way to be a teacher."

While a lack of discussion of pedagogical tools was generally found within the data, in a variety of facets, so too was the lack of faculty knowledge.

Both groups of faculty generally discussed a lack of knowledge regarding the use of music as a potential structural barrier. This lack of knowledge regarded how faculty; 1) may not understand "how it fits into a perspective, or a theory, or an idea," 2) may have a "lack of knowledge about how effective it is," 3) "may not see it as applicable," 4) may be unsure of "how to apply it in the classroom," 5) may not be knowledgeable about “current music trends," or, 6) may have "never thought about it" before. One DN faculty member illustrated how a lack of knowledge may hinder the use of music as a pedagogical tool during our conversation:

"M: How do you feel the use of music in college sociology courses might impact learning?

R: I don't have any idea, I never thought about it. Depending on the music perhaps, music connected to the definition of culture, or some topics, or it depends on the music probably. I haven't really thought about 
it. For me I don't think it would be useful. It would be competing tools with the visual image tools.

M: So you would see music taking away from visual image time or just competing with it, how so?

R: Competing in time for the structure of the class. I never thought about it. It could be a useful tool too if we know how to instrument the proper music for each topic.

M: So, you're saying maybe if there was greater knowledge about which songs go well with which concepts, that could help, it would be more useful?

R: Yes, but I don't have that knowledge."

Other faculty members also perceived music as beneficial and described a lack of knowledge or access to resources. As another DN group faculty member described:

"I'm a little bit older, I think if somebody is more knowledgeable about current music trends they would be able to use it more effectively. The bands that I know are 15, 20 years old and won't probably sustain the interest of students....If I came across some books or articles that showed me how I could do it, I would."

Here it is shown how faculty who did not use music in their courses may be open to learning more about the technique. Also found within the above quote is a concern about not being knowledgeable about current music trends as it related to one's age. 
Faculty members in both groups (and especially the DN group) discussed age when identifying a lack of knowledge of current popular music as a potential barrier. As a younger $\mathrm{DN}$ group faculty noted:

"I'm not as hip as I was though when I started, so I don't know what is considered popular any more. I think I would be slightly apprehensive in being able to pick music that is both popular enough, that it's well known, and that can convey the message that I'm trying to portray."

This sentiment was also shared by other faculty members who have used music on a less consistent basis in their past sociology courses. Another DN group faculty member mentioned:

"I did also learn an important lesson in pop culture this last time, I guess I'm getting old because at the end of the semester one of my students wrote in the eval; 'the class was great, it was really fun, but she needs to update her music references. None of us know who those people are.' I was like, you don't know who Bruce Springsteen is? So I did get a feeling in a couple of cases, it wasn't as effective as it should have been because the students, instead of being like oh yeah, they were like who, what?...I think it can be really helpful and engaging, but you also have to think about not letting it just seem old. You've probably had that experience where your instructor says something and you're like what decade are you living in? Then you realize that you're there now, but the bands I love are vintage." 
The lack of knowledge of popular music, and the lack of connection it may create with students, was also expressed by other faculty members as well. Another DN group member discussed:

"for me, in my intro class it would be very hard for me to find [music], and sometimes I actually do relate songs, and they don't necessarily know what I'm talking about. It's getting to the point where my Skater Boy by Avril Lavigne reference is not known anymore."

The lack of knowledge of music and the potential lack of connection with students will be explored further in the discussion chapter. The last similarity found between both groups of faculty members regarding potential barriers is the use of music being viewed as not common practice.

Both groups of faculty members generally viewed the use of music as an uncommon practice in higher education sociology courses in the United States. ${ }^{45}$ Most participants "guesstimated" that music is used as a pedagogical tool by less than twenty percent of faculty members in the U.S. ${ }^{46}$ When asked to speculate as to why only "X percent used music as a pedagogical tool, faculty discussed a varying degree of potential explanations that are either incorporated into other potential barriers/costs or were not found to fit in with any particular theme. ${ }^{47}$

\footnotetext{
${ }^{45}$ One faculty member from each group either stated they "didn't know" or were undecided, and another DO group faculty member believed music to be used by half of sociology faculty members, the rest of the participants believed the use of music to be not common among sociology faculty members in the U.S.

${ }^{46}$ Three faculty members from the DN group did not offer a guesstimation, and the majority of the faculty members who did offer a guesstimation believed music as a pedagogical tool to be used by $<20 \%$ of sociology faculty members in the U.S.

47 Although some explanations were found not to fit in with a common theme, it is worth mentioning how some faculty members believed the use of music may take "a lot of work to find the best songs," or a
} 
How the use of music being viewed as an uncommon practice by faculty members may serve as a potential barrier will be discussed further in the next chapter, when relating these findings back to Mead's theory. Beforehand, I turn to discuss the key differences found between the two groups of faculty members regarding the potential structural barriers associated with the use of music as a pedagogical tool.

\section{Key Differences}

Three key differences existed between the faculty groups regarding how they understood potential structural barriers. DO group faculty members generally discussed two additional potential barriers related with the use of music as a pedagogical tool in higher education sociology courses (i.e. negative views of colleagues and technological/copyright issues). DN group faculty members generally mentioned a lack of value placed on teaching as a potential barrier when discussing other pedagogical tools, including the use of music.

The first difference found between the groups is how DO group faculty members generally identified negative views of colleagues as a potential barrier for the use of music. DO group faculty members believed the use of music may be viewed by colleagues as "gimmicky," "frivolous and silly and a little weird," "less academic," "not useful," or as a "distraction." One DO group faculty member described:

\footnotetext{
"laborious task to do." Other faculty members mentioned how "faculty take themselves so seriously," how some institutions have a "puritan ethic" regarding student learning, how "we still rely on texts," or how there may be only a "relatively small proportion [of sociology faculty members] that happens to be wanting to do something really engaging."
} 
"Someone might judge [using music] as entertainment, someone might judge that as gimmicky, the same way as I made a judgment earlier on someone else's teaching...I think some of our peers and colleagues are maybe a little reluctant to use the word fun in the same sentence as learning...I say, expletive that. Who decided learning can't be fun or can't at least have a fun component. It doesn't make it entertainment, it doesn't degrade it, it doesn't cheapen it."

Other faculty members discussed how the use of music, along with other popular culture references, may be viewed as inferior and "not seen as something that can be intellectual or academic.” Another DO group faculty member noted:

"I think that the study and analysis and subsequent use of pop culture is sort of low, it's low brow basically. The university is considered this high brow institution of higher learning and education, and quite frankly songs like "Fuck the Police" just don't belong in the university...I think that there's a lot of people who look down upon the use of music and pop culture because it takes a little creativity. Somebody like Justin Bieber for instance, I don't like him at all but there are a lot of people who do, so perhaps we could use it to demonstrate course concepts, but that requires a little bit of thinking because I'm not a fan of his music but perhaps I can figure out a way to make this work in the classroom and to get people engaged. I think there's a lot less of that because aesthetically it's not on par with the vision that people have of what a university professor 
does... university professors listen to jazz music, or classical music, this would be a more high brow demonstration of music as it were, but Mozart in the policing course, how do we make that relevant. I'm not so sure, maybe there is a way of doing it. I hadn't really thought about it, but the kids aren't really typically listening to Mozart these days. They're listening to other things."

DO group faculty members who discussed music as being potentially viewed negatively by colleagues, frequently did so while also citing age as a potential factor as well. As illustrated in the following statements of four DO group faculty members; "I think perhaps older faculty, not to stereotype, but may be less likely to take it seriously as a pedagogical tool."

"I work with a lot of people who are older and sort of disconnected, and I think they're less interested in music. To be clear, that's not to disparage my colleagues at all. They just have a different style and a different approach. Pop culture is not their thing. They typically don't use music, and I sort of have that reputation on campus, whereas they don't."

"Without sounding ageist, I think that there is kind of a changing of the guards of sorts. As the boomer generation ages out and as there's an increase in youth and youthful instructors, boy that sounds horrible, but 
younger professors, I think, and also those that are more technologically savvy, I think that's where you see it."

"By the way, I have to say, in the past though I think they thought it was frivolous and silly and a little weird, but that was real old school people and all those people are gone. My colleagues now are like, 'oh wow cool, great conceptual tool.' They wouldn't even question something like that, and they certainly wouldn't question me as a teacher."

The above statements are exemplary of how DO group faculty members commonly believed the use of music may be viewed negatively by older "disconnected" faculty members, while the last quote illustrated how the use of music may be viewed positively by colleagues. This will be discussed further in the following chapter.

The second difference found between the groups is that technology and copyright issues may serve as a potential structural barrier. DO Group faculty members generally discussed how technology or copyright issues may serve as a potential barrier for using music as a pedagogical tool. DO group faculty members cited how technology frequently fails. One DO group faculty member noted:

"And with technology, something always goes wrong. Technology is always an issue, and I don't want to be tech support for my students at all. So that, if you don't have a good multimedia center like I have access to, I think it would be very difficult." 
Another DO group faculty member described how failing technology may deter others from incorporating music or other forms of technology into their courses, stating: "sometimes of course the technology fails, something is broken, something doesn't work, something doesn't turn on, and there's not necessarily that support you need in the moment to get through it...it's tough, and by the way, there's a good answer for why you might not use technology; music, YouTube, whatever. It's like, If this shit's not going to work why I am going to use it? What the hell man, this is not easy standing in front of a group of 20 year olds and trying to be interesting and trying to keep them engaged. It's not going to help my cause to flub with technology and look like; 'look at this guy, this guy can't even get the technology working. This guy's an asshole.' It might not be that harsh...so, how eager are you to put yourself in that position?" And yet another DO group faculty member discussed how changing technological devices and copyright laws may inhibit the use of music in sociology courses, stating: "Technology is really my enemy. It is changing way too quickly, and honestly, I do not have the kind of money to buy things that I already own on tape. This is something that infuriates me about the media technology in the first place. I have a lot of stuff that I still have on VCR tapes, why should I have to buy this again on CD or DVD? They're taking the technological devices that would allow me to keep on doing this away, 
because I'm a dinosaur, obviously, and they're not making it possible for me to copy stuff over."

While DO group faculty members discussed technological/copyright issues and negative faculty views as potential structural barriers, DN group faculty members described another potential barrier for incorporating pedagogical tools into higher education sociology courses.

The third difference found between the groups of faculty members is that DN group members generally cited a lack of support or value placed on teaching as a potential barrier. DN group faculty members generally discussed a lack of value placed on teaching when describing; 1) potential barriers related with their own preferred pedagogical tool, 2) whether their department or institution encourages or discourages certain pedagogical tools, or, 3) why the use of music is not common in higher education sociology courses. One DN faculty member noted:

"I think the state of teaching in higher ed. is honestly abominable. I don't think we put that much emphasis on becoming really great instructors...I think a lot of people do not take great teaching nearly as seriously as they think about their research... and it's not that they don't care about teaching but for various reasons either aren't pushed to do that, or tenure is almost entirely based on your research, so as long as you're teaching is not horrible. There's not a lot of institutional support for thinking really hard about how to be a great instructor." 
In the following chapter I will discuss how some faculty chose to overcome some of the above potential barriers. First, I would like to briefly mention another potential barrier the data illuminated but was not explicitly described as a barrier.

The last potential barrier, that may or may not be associated with the use of music as a pedagogical tool, is faculty autonomy. DO group faculty members generally discussed faculty autonomy when describing whether their department or institution encourages or discourages certain pedagogical tools (just under half of DN group members also discussed faculty autonomy in a similar fashion). While faculty autonomy was not specifically discussed as a barrier, it could potentially be related to the lack of discussion and knowledge associated with the use of music as a pedagogical tool found in this study. This will be discussed further in the next chapter, after a general summary of the findings is provided.

\section{General Summary of The Findings}

In this chapter, the findings of this research were presented. It was established that both groups of faculty members believed student engagement can lead to increased student learning, while they perceived music (along with other pedagogical tools) as being potentially effective in promoting student engagement and learning in higher education sociology courses. All faculty members generally agreed that music as a pedagogical tool may be beneficial in; 1) promoting student engagement, 2) illustrating sociological concepts, theories or ideas, and 3) sparking the sociological imagination (i.e. 
through connecting content with students' individual lives). Both groups of faculty generally incorporated discussion, writing assignments, lecture, film, PowerPoint, and other pedagogical tools into their courses. ${ }^{48}$ DO group faculty members generally incorporated music into their courses. With regard to other pedagogical tools, DO group faculty members generally discussed how they can be used to connect the content to the individual lives of students, while DN group faculty members generally described how other pedagogical tools related to encouraging student engagement. ${ }^{49}$ DO group faculty members generally identified two additional benefits potentially associated with the use of music as a pedagogical tool (i.e. music may encourage discussion and have other physiological benefits) along with a potential cost (i.e. faculty "may miss the mark"), none of which $\mathrm{DN}$ group faculty members mentioned.

Both groups identified, or illustrated, how a lack of faculty discussion of pedagogical tools and a lack of faculty knowledge regarding the use of music existed as potential structural barriers that might impact faculty choices to use music as a pedagogical tool. Both groups of faculty also viewed the use of music as a pedagogical tool in higher education sociology courses as an uncommon practice. DO group members generally identified two additional potential barriers associated with using music as a pedagogical tool (i.e. negative views of colleagues, and technological or copyright issues). DN group members generally identified a lack of value placed on teaching as an additional potential barrier for incorporating other pedagogical tools (as

\footnotetext{
${ }^{48}$ Some of which are related with the active learning approach (e.g. activities, games, discussion or writing assignments).

${ }^{49}$ Note: both connecting students with the content and engaging students are both educational outcomes related with the active learning approach.
} 
well as music). DO group faculty members generally discussed faculty autonomy as being encouraged by departments or institutions (e.g. "do your own thing"), but did not explicitly discuss it as a potential barrier associated with the use of music as a pedagogical tool. The next chapter will focus on discussing these findings, particularly with regard to the literature presented in Chapter Two. 


\section{Chapter Five: Discussion}

This chapter is organized into three sections. First, key findings and new understandings will be discussed. Second, the findings will be applied to the theoretical framework of this study. Finally, the relevance of the findings will be illustrated through revisiting the reviewed literature.

\section{Key Findings}

There are three key findings from this research. The first key finding is how the lack of discussion of pedagogical tools found among faculty members and in completed teaching courses may serve as a potential barrier for understanding how colleagues use music, and other pedagogical tools, in their sociology courses. One unintended consequence of this lack of discussion may be a misunderstanding of the potential benefits, costs, or barriers associated with the use of music as a pedagogical tool.

In this study, DN group faculty members were generally less knowledgeable than their DO group peers about the use of music as a pedagogical tool in higher education sociology courses. ${ }^{50}$ More specifically, DN group members lacked knowledge regarding; 1) how music may be used to encourage classroom discussion, 2) the physiological benefits of using music, 3) the potential costs, and 4) how to overcome barriers associated

\footnotetext{
50 As stated earlier, DN refers to faculty participants who did not use music consistently in their courses, whereas DO refers to faculty participants who did.
} 
with the use of music, such as how a lack of knowledge of current music trends may be alleviated through involving students in the song selection process. One possible explanation for DN group members' apparent lack of knowledge may be because they, when compared to DO group faculty members, were less likely to have completed a teaching course as part of their graduate school experience. However, this explanation seems unlikely because many DO group faculty members noted a lack of discussion of pedagogical tools and approaches in the teaching courses they completed in graduate school. The most likely explanation for DN group members' lack of knowledge of music as a pedagogical tool is the general lack of discussion of pedagogical tools and approaches found amongst sociology faculty as a whole.

The second key finding is that $\mathrm{DN}$ group faculty members did not view music as a pedagogical tool negatively. When provided the opportunity to discuss and think about the technique, DN group members in this study were open to the use of music as a pedagogical tool. Both $\mathrm{DO}$ and $\mathrm{DN}$ group faculty members identified multiple potential benefits associated with the use of music in higher education sociology courses. Specific benefits noted by both groups of participants included promoting student engagement, illustrating concepts, and sparking the sociological imagination. Interestingly, many DN group faculty members indicated they might use music in subsequent courses. When asked if they would "be likely to use music as a pedagogical tool in [their] sociology courses in the future," six out of the nine DN faculty members stated they would to some degree. ${ }^{51}$ For the DN group faculty members who stated they would not be likely to use

\footnotetext{
51 Only three DN group faculty members reported using music on an inconsistent basis.
} 
music as a pedagogical tool in the future, a lack of knowledge regarding how to select songs that are "both popular enough" and relevant for illustrating sociological concepts was their primary concern. Most of these faculty members also mentioned how they might be open to using music in their courses in the future if they were provided with the appropriate resources, such as books, articles, or "knowledge."

The third key finding is how unreliable technology and copyright issues, rather than a lack of access to audio equipment, are now seen as the greatest potential technology-based barriers for the use of music as a pedagogical tool in higher education sociology courses. DO group faculty members were much more likely to describe failing technology or copyright issues, as opposed to not having access to the appropriate resources to play music, as potential barriers for the use of music as a teaching tool. This may be due to an increase in access to high-technology classrooms; where technological devices such as computers, projectors, and speakers are provided by the institution. With regard to specific technological issues, most DO group faculty members discussed how sometimes technology fails; such as computers have problems, speakers don't work, or music doesn't play. Other consistent music users explained how it is becoming more difficult to either copy music from an older format to a newer one supported by their school, or to post songs to their online learning management systems such as Blackboard or D2L. To overcome potential copyright issues some faculty members just put "fair use doctrine" on their syllabi and "move on," while others have to abide by more strict guidelines when using music in their courses. For example, one DO group faculty member noted that copyright laws with regard to higher education were still being 
defined by the Canadian courts, so some faculty members may have been fearful to use music or film in their courses due to potential copyright infringement.

\section{Findings in Relation to the Theoretical Framework}

As discussed in Chapter Two, Mead (1934) identified how shared meaning is created through social interaction and shared significant symbols. It is through social interaction where shared symbols are both created and legitimated by others. These "others" may be another individual, or a group of individuals that constitute the "generalized other" (i.e. a social group one identifies with). A faculty member's department, institution, or discipline may serve as generalized others, from whom faculty members learn what is considered effective pedagogy through creating meaning with colleagues and other higher education professionals. Without social interaction, shared meaning is neither created nor legitimated. The shared meaning found amongst the participants in this study may also be shared by similar sociology faculty members in similar institutions.

In this study, it was expected that individual DN group faculty member's perceptions of the legitimacy of music as a pedagogical tool would have been shaped by the views of other faculty members, and that most other faculty members' views would have been negative. In Mead's terms, individual interactions with other faculty members who mainly held negative views about the use of music as a pedagogical tool would result in DN group faculty members developing a shared meaning that music was not a 
legitimate pedagogical tool and this would account for why DN faculty members did not use music in their sociology courses. This turned out not to be the case. Instead for DN faculty members in this study, their lack of use of music in their courses was due to a lack of interaction with knowledgeable others. Most DN faculty members, rather than viewing the use of music negatively, never discussed it with other faculty members and therefore did not have a meaning assigned to the use of music as a pedagogical tool. During the interviews for this study, DN group faculty members were initially given time to reflect on and discuss their own educational background and the pedagogical tools they used in their courses. They were then given opportunities to think about and discuss the use of music in higher education sociology courses. Through this process DN group faculty members generally connected the use of music to their own experience, articulated potential benefits and speculated about potential barriers associated with the use of music as a pedagogical tool. ${ }^{52}$ Through the interview process, DN group members developed a set of shared meanings regarding the potential benefits of using music as a pedagogical tool and the potential barriers to its successful use in their courses.

Interestingly, DO group faculty members attributed a different motivation to explain why DN faculty members did not use music in their courses. DO group faculty members generally agreed that the negative views of colleagues were a potential barrier impacting faculty members' choices to use or not use music as a pedagogical tool. When discussing potential negative faculty members' views, DO group members cited how

\footnotetext{
52 It is also at this point (i.e. when I began asking questions regarding the use of music) where some faculty members began to question the focus of this study (e.g. "you're interested in music"), and later were able to ask clarifying questions of the researcher. In an attempt to avoid bias, when inquired I would discuss how this study is interested in the benefits, costs, and especially the barriers associated with pedagogical tools in general-including the use of music.
} 
music may be viewed as "frivolous," "silly," "weird," "a distraction," "gimmicky," "entertainment," "low-brow," or may not be taken "seriously as a pedagogical tool" by other faculty members who did not use the technique. Many of these discussions were speculative in nature, where faculty stated how they "thought" music was viewed negatively by faculty without citing direct experiences or interactions with other faculty members. While this claim was not corroborated in this study, Mead's theory would suggest that DO group faculty members developed a shared meaning for why DN faculty members may not use music as a pedagogical tool without ever discussing the technique with their colleagues. If DO group faculty members do not discuss the use of music as a pedagogical tool with their colleagues, then shared meaning cannot be created and the technique is not legitimated through social interaction with other faculty members. This lack of legitimization through interaction with others may lead DO group faculty members to believe the use of music is not viewed as a legitimate pedagogical tool by their colleagues - when in reality, colleagues may view it positively if given the time and opportunity to discuss the technique. This same theoretical framework may also be applied to further understand how a lack of interaction between faculty and students may lead to a lack of shared meaning regarding the use of music as a pedagogical tool in the classroom.

In this study, DO group faculty members described how students may view the use of music as "stupid," may misinterpret the song, or resent song selections. Past researchers described how faculty members may overcome this cost through "setting the stage" and being "explicit" when using music as a pedagogical tool. Through being 
explicit and explaining to students why music is being used, what it is seeking to accomplish, and how it is important for illustrating sociological concepts, faculty may create a shared meaning with students where the use of music can be viewed by both parties as a legitimate pedagogical tool when used in the classroom.

Mead's theoretical framework is also useful in understanding why both groups of faculty generally believed the use of music to be an uncommon pedagogical technique. Once again, for Mead, what is important is that faculty members do not discuss music as a pedagogical tool with their colleagues or in completed teaching courses. If a faculty member does not discuss the use of music with colleagues, or does not observe it being used in other faculty's courses, she/he may view music as an uncommon pedagogical practice-even though it may be more common than he/she believes. The lack of interaction-based shared meaning and legitimization of music as a pedagogical tool amongst higher education sociology faculty members, as well as in completed teaching courses, may lead music to be viewed as an uncommon practice. This lack of shared meaning may further serve to perpetuate group members' inaccurate beliefs regarding the use of music as a pedagogical tool in higher education sociology courses. As Mead noted, shared meaning and legitimacy can only be gained through social interaction. 


\section{Findings in Relation to Reviewed Literature}

\section{Potential Structural Barriers}

Reviewed literature identified several potential structural barriers associated with the use of music as a pedagogical tool, including; 1) a lack of value placed on teaching, 2) negative views of colleagues, 3) music viewed as an uncommon practice, and 4) a lack of access to proper equipment. The barrier of a lack of access was discussed thoroughly in the "key findings" section of this chapter. This section will relate the findings and data from this study to the other potential barriers from the literature.

DN group faculty members generally discussed a lack of support or value placed on teaching as a potential structural barrier that may impact faculty choices to use music as a pedagogical tool. While this barrier was also mentioned by a couple DO group faculty members, DN group members discussed the issue in greater detail. DN group faculty noted how "there is a lack of incentives to be an effective teacher," where "a lot of [faculty] do not take great teaching nearly as seriously as they think about their research.” Similar to Atkinson (2001), one DN group participant noted that "more effective teachers are being almost more punished than rewarded because that valuable time you spend giving feedback is time that takes away from writing articles and applying for grants." These types of comments are also similar to Rau and Baker's $(1989: 163,172)$ insight that "faculty are neither trained, motivated, nor rewarded for providing high quality instruction," which leads pedagogy to "never receive the time, effort, and creativity it requires." The lack of incentives and value placed on teaching 
may be a salient point when seeking to explain why a lack of faculty discussion about the usefulness of pedagogical tools and pedagogy exists. If, as Becher (1989) points out, academics gain prestige and credibility through research, and excellence in teaching counts little toward recognition from established colleagues, then it follows that faculty members may be more likely to discuss research-related topics rather than pedagogy with colleagues. A potential explanation for why a lack of value placed on teaching was only discussed by DN group faculty members may be due to the scholarship of teaching and learning movement's (Chalmers 2011) increased emphasis on the importance of teaching. That is, DO group faculty may believe awards, grants, and promotion programs have increased the value placed on pedagogy, but still project a negative view of the importance of music as a pedagogical tool onto their colleagues.

Although it was not supported in this study, literature from the early 1980s through the late 90s suggested negative views of colleagues may serve as a potential barrier for the use of music as a pedagogical tool in higher education. Cooper (1982) described how using music as a pedagogical tool may invoke "belittling faculty responses," while others warned that faculty "may face resistance and skepticism from [their] colleagues when [they] try to use music as a legitimate teaching technique" (Walczak et al. 1989:5). Pichaske (1999:92) attempted to explain this skepticism by stating that; "perhaps academics fear anything popular because it opens the possibility that students will know more about the subject than they." Even though DO group faculty members in this study generally identified negative views of colleagues as a potential barrier for the use of music as a pedagogical tool, DN group faculty members 
attributed their lack of use of music in their courses as due to a lack of interaction with knowledgeable others such as when they discussed a lack of knowledge of current popular music trends as a potential barrier, due to being "older" and "not as hip" as they used to be, but if they had the knowledge of how to keep music current (e.g. through involving students in the song selection process) they would use music as a pedagogical tool in their courses. The age of faculty members was also discussed by DO group members when speculating why only a small number of sociology faculty use music in their courses. While it was not supported in this study, DO group faculty members believed that older faculty members may be "less likely to take [the use of music] seriously" as a pedagogical tool, or may be less "technologically saavy" than younger faculty members. ${ }^{53}$ It may be the case that DO group faculty members have experienced these negative responses from colleagues in the past, and this might explain why they may see negative views of colleagues as a potential barrier; whereas, DN group faculty members have not had these experiences. This does not seem to be the case due to most DO group faculty members not citing direct experience when discussing negative views of colleagues as a potential barrier. A more fitting potential explanation was discussed in the theoretical section of this chapter. Perhaps because of the lack of discussion and legitimization of music as a pedagogical tool with colleagues and in completed teaching courses, users of music may feel that non-music using faculty members view the technique as illegitimate due to no collective meaning or legitimacy being assigned to the

\footnotetext{
53 As shown in the methodology and findings section, DO and DN group members are fairly similar in age. Although two older faculty members are in the DN group, when compared to the DO group, DN group members commonly viewed the use of music as potentially beneficial and were open to using it. When discussing how he could have used a Woody Guthrie or Chamillionaire song in one of his courses, one older DN faculty member stated "I probably could have pulled it up on YouTube as a matter of fact."
} 
use of music as a pedagogical tool through social interaction. One DO group faculty member chose to overcome perceived negative faculty views through publishing about the usefulness of music as a pedagogical tool. He/she noted that; "as soon as I started publishing and teaching sociology about it, then it was considered acceptable." In the future, potential negative non-user faculty views may be alleviated through greater faculty discussion and dissemination of information regarding the usefulness of music as a pedagogical tool. As Hinds-Aldrich (2012:481) stated; there is a "small but growing body of literature advocating the use of nontraditional academic media," including the use of music, which may help these tools gain greater legitimacy among sociology faculty members in the future.

Finally, past researchers briefly mentioned that the use of music as a pedagogical tool may be viewed as an uncommon practice. This point is supported in this study as well. While the literature does not provide accurate information on the actual percentage of faculty using music as a pedagogical tool in higher education sociology courses, faculty in this study generally "guesstimated" that music is used by less than twenty percent of higher education sociology faculty in the United States. This suggests that faculty members in this study believed the use of music as a pedagogical tool to be an uncommon practice amongst higher education sociology faculty members. Regardless of whether the speculated percentage is accurate or not, this study's finding that faculty rarely engage in pedagogical discussions with colleagues suggests that the perception of a low usage of music amongst faculty members may be influenced more by the lack of pedagogical discussions than based on accurate music-usage data. It may be the case that 
the use of music is more common than participants thought. However, since the technique is not commonly discussed, faculty may not be knowledgeable about who does and who does not use music as a pedagogical tool in their courses. ${ }^{54}$

Potential Costs

DO group faculty members identified one potential cost of using music as an instructional tool, stating that faculty may "miss the mark" when using the technique. "Missing the mark" includes students potentially resenting song selections, becoming 'emotionally stressed,' choosing music that illustrates negative stereotypes, or believing it is ok to use inappropriate language. Similar to earlier studies by Walczak and Reuter (1994) and Hinds-Aldrich (2012), DO group faculty in this study discussed how students may not enjoy the type of music being played or be able to connect with a particular song as a popular culture reference. One DO group faculty member noted; "if you don't have good reasoning behind why you're using [a particular song], students might react negatively to it, see it as a waste of their time or this guy's crazy, or something like that."

Similar to Martinez (1998), DO group faculty members in this study also discussed how certain songs may offend or stress students. Some DO group faculty chose to overcome the potential cost of offending students by not playing "risky" music. This finding is consistent with Palmer's (2011) point where he invested in censored versions of songs, or did not play a song if it felt "too risky."

\footnotetext{
54 This is applicable to other pedagogical tools as well, since in this study it was found that faculty members did not discuss pedagogical tools in general.
} 
Similar to Munkres (2010), one DO group member cited that white "privileged" students may misinterpret the music and select songs that replicate stereotypes and negativity. Interestingly, another DO group faculty member in this study raised a related point that was not found in the reviewed literature by noting that students, after listening to contemporary rap and hip-hop music chosen by the faculty member to make a pedagogical point, may think it is ok to use inappropriate, misogynistic, or "derogatory" language. This faculty member discussed overcoming this potential cost, by stating; "I've got to be vigilant about emphasizing the purpose for using these songs or for what I'm trying to accomplish here...the danger would be subtly sending the message that you can use this language." This resembles an earlier point made by Hinds-Aldrich (2012) and Martinez (1998) where they described the need for faculty to 'set the stage' and explain how the music specifically relates to the content prior to playing a song.

Finally, similar to Martinez (1994), regardless of the potential costs associated with 'missing the mark,' DO group members in this study explicitly stated that they believed the potential benefits of using music as a pedagogical tool outweigh the potential costs.

\section{Potential Benefits}

In this study, both groups of faculty members generally mentioned promoting student engagement, illustrating concepts or theories, and sparking the sociological imagination as potential benefits associated with the use of music as a pedagogical tool. DO group participants noted two additional benefits. First, music can be an effective tool 
for encouraging classroom discussion. Second, music may provide physiological benefits for students as well. All of the benefits discussed in this study have previously been identified by other researchers.

Similar to Ahlkvist (1999), Elterman (1983), and Hinds-Aldrich (2012), faculty members in this study believed music can be used as an effective pedagogical tool for promoting student engagement. It appears this finding may be helpful with regard to Hill's and others' (2012) suggestion for future research. It appears since DO group faculty members commonly used both PowerPoint and music as pedagogical tools in their courses, that music may be embedded into slides to help make PowerPoint an effective tool for engaged learning. ${ }^{55}$ What is particularly unique to the current study is that DN group faculty agreed that music could be used effectively to increase student engagement, even when they had not previously given much thought to using the technique or when they considered themselves as unknowledgeable or incapable of integrating music into their own courses.

In this study, both groups of faculty also agreed that music may be beneficial for illustrating sociological concepts, theories, or ideas. Similar to Ahlkvist (2001), DO group faculty members were more likely to discuss using music to illustrate sociological theories than DN group faculty. On the other hand, DN group faculty members were

\footnotetext{
55 In my own personal attempts at embedding audio into PowerPoint slides, it was at first a very difficult task due to failing high-technology classrooms (e.g. speakers not working) and a lack of understanding of how to properly embed audio files so they can play when cued on different computers. But after many trial and error attempts and Google searches, I can now use music in PowerPoint slides quite easily (although it is still a good idea to test the presentation and $\mathrm{a} / \mathrm{v}$ equipment when working in an unfamiliar classroom before students arrive).
} 
more likely to discuss how music may be used to illustrate specific concepts such as deviance, similar to Bach and Albers (2010) and Munkres (2010).

Similar to Burns and Martinez (1993), faculty from both groups in this study identified that music may be used to "spark" the sociological imagination. This finding is consistent with previous research (Bach and Albers 2010, Boggs 1993, Elterman 1983, Martinez 1995, Munkres 2010, Walczak 1993, Walczak et al. 1989) that found the use of music as a pedagogical tool helped students to create a connection between the concepts and their individual lives. Also similar to Burns and Martinez (1993) and Albers and Bach (2003), some faculty members in the current study discussed that music may be used to create a connection between sociology faculty and students. As one DO group faculty member described; "music helps me, I think, have that slice of credibility [with students] that is required in order to pass on some very complicated and difficult ideas." Also, as one DN group faculty commented regarding her observation of a colleague using music; "it was a connection to the topic, it was a connection to the instructor, and...it was a connection to culture."

DO group faculty members generally identified the potential benefit of using music to encourage classroom discussion, similar to the findings of Martinez (1995) and Palmer (2011). As one DO group faculty member stated; "whatever I have to do to pull them into the discussion I will do, as long as it's not idiotic and unethical, or outright stupid. I think a song, in my view, is a pretty safe way to pull students into the discussion." This is similar to another DO group faculty member that described how music "kind of breaks the ice, [and] leads into a nice discussion about the topic." 
The final potential benefit identified by DO group faculty members involved the potential physiological benefits associated with the use of music as a pedagogical tool. Similar to Ahlkvist (1999), Elterman (1983) and Keskin (2011), DO group faculty members in this study discussed how using music may help "alleviate anxiety" and create a more "relaxing," "comfortable," "memorable," "interesting," and "fun" learning environment. Reminiscent of Palmer (2011) and Walczak and Reuter (1994), DO group members in this study also described how students" "faces light up," or "perk up," when using music as a pedagogical tool in their sociology courses.

\section{Active Learning}

In this study, both groups of faculty agreed that student engagement can lead to increased student learning in higher education sociology courses.

As discussed in the review of the literature, there are two important aspects of the active learning approach: 1) incorporating engaging activities into the classroom to help break up the lecture and keep students "actively engaged," and 2) connecting content to what students already know (i.e. their individual lives and existing knowledge base). In this study, faculty members from both groups mentioned the above aspects of the active learning approach. DO group members, when discussing other pedagogical tools, were more likely to focus on the importance of connecting content to the individual lives of students. DN group members in this study believed other pedagogical tools may increase student engagement, but did not generally agree on any other potential benefits (such as connecting content to the individual lives of students). 
In their study, Andrews et al. (2011) found that while the majority of faculty incorporated engaging activities into their courses, activities alone do not lead to increased student learning. Similar to the active learning approach, Andrews et al. (2011) discussed the importance linking content to the existing knowledge base of studentswhich they found to be lacking in most faculty members' understandings of the active learning approach. ${ }^{56}$ Relating these findings back to this study, it may be that DO group faculty's greater focus on the importance of connecting content to the individual lives of students may be due to a better understanding of the active learning approach. This difference may be due to DO group faculty members' greater likelihood of having completed a teaching course while in graduate school, even though faculty in this study reported that pedagogical approaches and tools were not commonly discussed in these teaching courses. A second possible explanation for this difference may be that DO group faculty members might be more involved in the scholarship of teaching and learning than $\mathrm{DN}$ group faculty members. ${ }^{57}$

In this study, both groups of faculty participants generally mentioned pedagogical tools related to the active learning approach. ${ }^{58}$ Most commonly faculty members identified using discussion (i.e. classroom, small group, or both) and writing assignments

\footnotetext{
${ }^{56}$ Andrews et al. (2011) also found a positive correlation between student interest in a course and student learning. This is important due to music being discussed as a way to increase student interest in sociology courses both in reviewed literature (Ahlkvist 1999, Elterman 1983) as well as in the data of this study. ${ }^{57}$ It also may be the case that DN group members generally believed that connecting content to students' individual lives is important, but they did not identify this as a general benefit because they were discussing the costs/benefits/barriers of different preferred pedagogical tools. Although possible, this still does not explain the difference found between the groups when giving an example from their own experience of how student engagement may lead to increased student learning - where DO group faculty members were more likely to discuss connecting content to students' existing knowledge base than DN group faculty members.

${ }^{58}$ Some examples of active pedagogical tools identified by Bean (2011) include discussion, writing, critical thinking activities, student as teacher, role playing, or simulation games.
} 
as pedagogical tools in their courses, which were both identified by Bean (2011) as being related to the active learning approach. Based on the findings of this study, music may be most beneficial for promoting both aspects of the active learning approach through encouraging students to discuss and analyze songs and lyrics as they relate to the course content.

In an earlier section of this chapter it was noted that both DO and DN group faculty members in this study generally viewed the use of music as beneficial for both engaging students and helping them connect sociological knowledge to their own individual lives. This finding demonstrates that both groups of faculty recognized that using music as a pedagogical tool can help promote the two main aspects of the active learning approach. ${ }^{59}$

\section{Building a Community of Learners Through Using Music as a Pedagogical Tool}

Chapter One posed a question regarding whether or not music might be used as a pedagogical tool to promote building a community of learners in higher education sociology courses. The findings from this study suggest the answer to this question to be "yes."

In this study, both groups of faculty members generally believed that music can increase student engagement in higher education sociology courses. Through using

\footnotetext{
${ }^{59}$ In this study, DN group faculty members lacked knowledge regarding how music may be used to enhance classroom discussion. This may deter faculty members from using the technique. That is, DN group members believed using music may increase student learning but did not know how to use it to effectively engage students in discussion (which discussion is one of the main pedagogical tools associated with the active learning approach, and it is through discussing songs and lyrics where music appears to be most beneficial in the classroom).
} 
music as a pedagogical tool, faculty members may develop an active role for students and create a common language of discourse through engaging students and creating opportunities to discuss songs and lyrics in relation to the course content. An example of this is found in Chapter Four, where one DO group faculty member described how music can get students to talk to one another and discuss their own music preferences, while following up with a broader discussion of the social forces that may help shape their own individual preferences. This is similar to Albers and Bach's (2001) example of how, through class discussion and sociological analyses of popular song lyrics, they were able to create a common language of discourse that was familiar to both students and faculty members alike.

Similar to Berk (2008) and Keskin (2011), DO group faculty members in this study identified that music as a pedagogical tool may be used to create a "comfortable," "relaxing," "lightened" and "fun" mood in the classroom environment. As one DO group faculty member stated; "I think a song, in my view, is a pretty safe way to pull students into the discussion." These findings appear to support the idea that music as a pedagogical tool may be used to promote a safe and supportive learning environment. When relating the findings from this study back to the suggestions provided by Macheski et al. (2008), it appears music as a pedagogical tool can be used to; 1) develop an active role for students by involving them in the role of social researcher through analyzing song lyrics for sociological content, 2) create a common language of discourse through collectively interpreting and using the artist's language to illustrate a theorist's concepts, and 3) build a supportive emotional environment through creating a 
comfortable, relaxing, fun, and safe environment. Similar to Maier (2013), it appears that DO group faculty members in this study also believed that using music as a pedagogical tool can assist in building a community of learners in higher education sociology courses.

\section{Summary}

This chapter reflected on the findings of this study. Three important findings were discussed. First, a lack of discussion of pedagogical tools among faculty members and in completed teaching courses may lead to a lack of understanding of the use of music, and of non-users' lack of knowledge regarding the use of music as a pedagogical tool. Second, non-music using faculty members may possess generally positive attitudes toward the use of music — something that was discussed very minimally in the reviewed literature. Finally, a shift may have occurred in what music-users identify as the major technological barrier to the use of music as a pedagogical tool. For music-users in this study, failing technology and copyright issues were harder to overcome than the issue of a lack of access to audio equipment. This chapter also discussed how the findings from this study relate to previous literature. The final chapter will discuss the implications and limitations of this study, and how the findings may inform future research. 


\section{Chapter Six: Conclusion}

The conclusion chapter will be divided into three sections. First, I will examine the implications of this study's findings for practice, theory, and knowledge. Next, I will discuss the limitations of this study. Finally, I will suggest how the findings from this study may inform future research.

\section{Implications}

\section{For Knowledge}

In this study, several new understandings were gained regarding the use of music as a pedagogical tool. First, faculty participants reported they did not commonly discuss pedagogical tools with their colleagues. Similarly, the teaching courses completed by participants during their graduate school experience did not commonly discuss pedagogical tools or approaches. One unintended consequence of this lack of discussion of pedagogical tools appears to be a misunderstanding among DN group faculty members regarding the potential benefits, costs, or barriers associated with the use of music as a pedagogical tool. In this research DN group faculty were less knowledgeable about the use of music as a pedagogical tool. This appears to be due to a combination of faculty not discussing the use of music and other pedagogical tools with their colleagues, as well 
as faculty not being exposed to discussions of the benefits and costs associated with different pedagogical tools in their graduate school teaching courses.

The second new understanding is that faculty members who do not use music consistently in their courses believed music to be a potentially beneficial pedagogical tool, and were open to using the technique in the future. This finding is contrary to those from previous research that identified colleagues' negative views of music as a potential barrier to faculty using music as a pedagogical tool in their courses.

The third new understanding gained is that faculty members who use music consistently in their courses identified technology failure and copyright issues as potential barriers to using music as a pedagogical tool, rather than a lack of access. This finding also contradicts findings from earlier research. This shift in faculty members' views of potential barriers associated with the use of music as a pedagogical tool may be the result of the increase in availability of high technology classrooms on college campuses.

The fourth new understanding is that faculty participants generally viewed the use of music in higher education sociology courses in the United States to be an uncommon practice. While there is no current data on the number of sociology faculty using music as a pedagogical tool, this finding corroborates similar findings from the reviewed literature. Faculty members" "guesstimates" of music-usage serve as indicators of underlying beliefs. Therefore, regardless whether the guesstimated percentage ${ }^{60}$ is accurate or not, this finding suggests that faculty members believed the use of music as a

\footnotetext{
${ }^{60}$ That is, the use of music as a pedagogical tool was believed to be used by less than twenty percent of sociology faculty members in the U.S.
} 
pedagogical tool to be an uncommon practice among higher education sociology faculty.

This finding is important because individuals' beliefs strongly impact their actions.

Faculty members who believe that colleagues are not using music as a pedagogical tool may be less likely to try and use it as a tool themselves. This finding also connects with the first finding from this section; that is, faculty members' guesstimations appeared to be influenced more by the lack of discussion of pedagogical tools with colleagues and in completed teaching courses than knowledge of the actual usage of the technique.

Finally, this study added information to the discussion of higher education sociology faculty members' usage of pedagogical tools. My review of the literature regarding pedagogical tools found little discussion of which pedagogical tools higher education faculty used most frequently. ${ }^{61}$ Participants in this study generally reported incorporating lecture, discussion, writing assignments, film, and PowerPoint into their courses.

\section{For Practice}

This study appears most useful for practice in discussing how to potentially alleviate prospective barriers and costs associated with the use of music as a pedagogical tool. More specifically, the findings of this research add to the discussions of two potential barriers associated with the use of music; 1 ) how to address faculty members'

\footnotetext{
61 This is applicable to other disciplines in higher education as well. Many researchers believed that straight lecture is the most frequently used, commonly citing it as a "traditional" pedagogical tool used by many faculty members, but they appeared to not have scientifically measured which pedagogical tools are used most often by higher education faculty members.
} 
lack of discussion and knowledge of pedagogical tools, and 2) how to overcome faculty members' concerns regarding "missing the mark" with students in their classrooms.

First and foremost, it appears that a more formal approach needs to be taken by sociology departments in order to overcome the lack of discussion and knowledge of music as a pedagogical tool found in teaching courses and amongst faculty members in this study. A more conscientious effort to incorporate discussions of pedagogy and pedagogical tools into completed teaching courses is needed. In this study, most faculty members completed a teaching course as part of their graduate school experience, ${ }^{62}$ yet most agreed that they did not discuss pedagogical tools and approaches in those courses. Clearly, one way to overcome this lack of discussion and knowledge of music may be to include more explicit discussions of pedagogical approaches (e.g. active learning) and pedagogical tools (e.g. the costs, benefits and barriers associated with using music) into future sociology graduate programs' teaching courses. It is also apparent that a more formal approach needs to be taken to increase faculty discussions and knowledge of the pedagogical tools of their colleagues. One way to do so involves setting time aside during mandatory faculty meetings to discuss and share pedagogical tools. ${ }^{63}$ In this way faculty members would not have to go out of their way, or create additional time, to increase their knowledge about pedagogical tools. Through a more formal approach to pedagogical discussions, non-music using faculty members may increase their

\footnotetext{
${ }^{62}$ Eleven out of sixteen participants completed a teaching course as part of their graduate school experience; that is, seven from the DO group, and four from the DN group.

${ }^{63}$ This would not have to be given much time. For instance, every meeting a different faculty member could give a brief presentation (e.g. 5-10 minutes) on a pedagogical tool they find to be effective for increasing student learning in their courses. This could be followed by a brief question-and-answer discussion (e.g. 5 minutes).
} 
understanding of the use of music as a pedagogical tool and music users may increase their comprehension of other pedagogical tools used by their colleagues.

Faculty members may also learn how to overcome potential barriers and costs associated with the use of music as a pedagogical tool through increased pedagogical discussions with their colleagues. In this study DN group faculty members who were not likely to use music in their courses in the future cited a lack of knowledge regarding how to select songs that are both popular enough with students and relevant for illustrating the content. These faculty members could learn how to incorporate students into the song selection process, if provided the opportunity to discuss and learn from their colleagues. One DO group faculty member noted; "I always invite [students]...give me an idea what you're listening to, tell me what songs matter to you, I'm not trying to be like the hip guy because if it's left up to me, I'm in a time warp, I'll be playing stuff from the 60s to the 90s...so you tell me what should we be listening to...to illustrate, convey, demonstrate, question, [or] explore [the content]." By reducing concerns about missing the mark with students regarding song choice, discussions between music-using and non-music using sociology faculty members could increase the chance of more sociology faculty members using music as a pedagogical tool in their future courses.

This study's final implication for practice involves drawing attention to the importance of faculty members properly setting the stage prior to playing music in sociology courses. Consistent with earlier research (Hinds-Aldrich 2012 and Martinez 1998), faculty members in this study described the importance of setting the stage by explaining to students how a song directly relates to the content prior to playing it. 
Proper set-up can help faculty members address concerns about "missing the mark" with students. Setting the stage may be helpful in several ways. First, this technique encourages students to view the song as an illustration of the content rather than as merely entertainment. Second, by explaining the academic relevance of a song, faculty members may mitigate students' reactions to any negative stereotypes or inappropriate language found within a song. Finally, setting the stage provides faculty members with the opportunity to create a safe emotional environment in the classroom for ensuing discussions of the music and lyrics. While not a panacea, it seems through properly setting the stage prior to playing songs in sociology courses, faculty members may alleviate potential issues of student resentment, emotional stress, or the perpetuation of negative stereotypes (or inappropriate language) within the classroom setting.

\section{For Theory}

This study validates Mead's theory regarding how shared meaning is created and legitimated. Faculty members learn what pedagogical tools are considered legitimate through interacting with their peers. In this study, the lack of shared meaning and perceived legitimacy with regard to pedagogical tools among participants appears to be based on a lack of interaction among sociology faculty members. This lack of interaction took the form of a lack of discussion about the strengths, limitations, and barriers of music as a pedagogical tool among faculty members and in completed teaching courses. The lack of discussion of music as a pedagogical tool among colleagues may have led DO group faculty members to misattribute the view that music is an illegitimate 
pedagogical tool to non-users. In fact, based on the data from this study, DN group faculty members held positive views of the use of music as a pedagogical tool. While Mead is clear that social interaction is necessary for the development of shared meaning with others, this study illustrates how a lack of interaction led to a lack of shared meaning and misunderstandings among faculty members. Although sociology faculty may interact with colleagues on a regular basis, if pedagogical discussions are not included in these interactions then potentially effective pedagogical tools may be overlooked due to faculty not creating a shared understanding of how these tools may be used effectively in the classroom.

\section{Limitations}

As is the case with social research, there are potential limitations associated with this study. First, it is important to note that the findings from this study are not generalizable in a probabilistic sense. This study did not draw a random sample of all sociology faculty members in the United States, and therefore the findings cannot be applied to the entire population of sociology faculty in the U.S. However, this study's findings may be transferable (or useful) to similar studies with similar questions of practice, or, to similar faculty located in similar departments and institutions (Marshall and Rossman 2011).

The second limitation of this study relates to the study's participants. The participants in this study were voluntary, and inclusion was dependent upon faculty 
responding to invitations via email or by telephone. It may be the case that faculty who chose to participate in this study have a stronger interest in the scholarship of teaching and learning, as opposed to other higher education sociology faculty members. If this is the case, the finding regarding DN group members' lack of negative views toward the use of music may be biased. Perhaps faculty members who do not use music in their courses generally view the technique in a negative fashion. However in this study, DN group faculty were unlikely to have completed a teaching course as part of their graduate school experience, while all DO group members reported completing such a course. What I cannot answer is whether DN faculty members' lack of completing a graduate teaching course was due to personal choice or a lack of opportunity in their respective graduate programs. If the lack of completing graduate teaching courses was based on individual choice, and DN group faculty members were more interested in research than in the scholarship of teaching and learning, then their positive views on music as a pedagogical tool may be shared by a relatively larger percentage of non-music using, researchfocused, sociology faculty members.

The final limitation to be discussed from this research involves the pedagogical tools that faculty members reported using in their own teaching experience. Faculty members in this study may have under-reported the actual number of pedagogical tools they used in their own teaching experience. As part of a completed course in adult learning and development, I recently had the opportunity to observe two higher education sociology courses and complete follow-up interviews with both faculty members. ${ }^{64}$ Both

\footnotetext{
64 Also of relevance, and similar to the findings of this study, is how both faculty members interviewed; 1) believed student engagement may lead to increased student learning, and 2) lacked knowledge regarding
} 
faculty members were observed using pedagogical tools and activities related with the active learning approach, and observed pedagogical tools were documented and counted. During the completed interviews, I had the opportunity to inquire about what pedagogical tools each faculty member believed he/she used in their own teaching experience. In this course assignment, both faculty members discussed using fewer pedagogical tools than were actually observed in their courses. While faculty members in this study generally incorporated lecture, discussion, writing assignments, film, and PowerPoint into their courses, it may be the case that they under-reported the range of pedagogical tools they used in their classrooms. While this may be the case, the interviews analyzed in this study were generally longer and more detailed than the interviews completed as part of the adult learning and development course assignment. Thus, faculty in this study had multiple opportunities throughout the interview to discuss the pedagogical tools they used in their own courses. For example, when discussing what pedagogical tools other colleagues may have used in their courses, or their own graduate school experience, faculty participants were occasionally reminded of pedagogical tools and discussed using similar techniques in their own courses.

the pedagogical tools used by their colleagues citing a lack of discussion of pedagogical tools with other faculty members, as well as during department meetings. 


\section{Future Research}

\section{How Pedagogical Tools Gain Legitimacy}

In this study, I found that higher education sociology faculty members in both groups generally incorporated lecture, discussion, writing assignments, film, and PowerPoint into their courses. Since the majority of sociology faculty members reported using these pedagogical tools, it seems fair to conclude that they are generally viewed as legitimate teaching tools. While the legitimization of lecture and discussion may be potentially explained through past exposure, ${ }^{65}$ how did the media-related pedagogical tools of film and PowerPoint gain legitimacy?

As noted earlier in this thesis, one way pedagogical tools may gain legitimacy is through social interaction with colleagues. As suggested by Hill et al. (2012), it may be through student expectations or institutional pressure to receive positive student evaluations where pedagogical tools gain legitimacy. Another way the legitimacy of specific pedagogical tools can be established is when faculty members make supportive arguments in scholarly articles. I found evidence of faculty members using this approach to establish the usefulness of music. One DO group faculty member explained; "as soon as I started publishing and teaching sociology about [music], then it was considered acceptable [by older faculty members]." On the other hand, another DO group faculty member discussed that "writing an article about the use of music in your classes is not going to get you anywhere." This same faculty member also elaborated that non-music

\footnotetext{
${ }^{65}$ That is both lecture and discussion were found to be generally incorporated into faculty members' graduate school courses.
} 
using faculty members may be convinced of music's potential usefulness through a teaching demonstration, but she questioned whether or not sociologists in the U.S. even value teaching. She stated; "if we value teaching, we would be more interested in adopting proven, good tools which do exist." This leads nicely to two potential questions for future research; 1) how do "good" pedagogical tools become viewed as such?, and 2) how do pedagogical tools become generally incorporated into higher education sociology courses? Through understanding how other "non-traditional" pedagogical tools have gained legitimacy in the past, it may be further understood how the use of music may gain greater legitimacy in the future.

\section{A Lack of Training in Pedagogy and Faculty Autonomy as Potential Barriers to} Pedagogical Discussions Among Faculty Members

In this study, numerous participants discussed faculty autonomy, usually in responses to the questions; "would you say some pedagogical tools are encouraged or discouraged by your department," “...or institution?” Faculty members from both groups discussed how "there is such a thing as academic freedom," where "nobody is going to breathe down your neck about how to teach." ${ }^{66}$ As one faculty member described; "we assume everybody has the professionalism to teach their courses as they should in a university." This appears to be a commonly held belief in higher education, even when faculty members have not previously completed a teaching course.

\footnotetext{
66 Numerous faculty participants discussed how faculty members are usually held accountable for their teaching practices through student teacher evaluations. One faculty member stated; "if it's good for you, and your students tend to be engaged and happy, then they're okay with that."
} 
As previously discussed, faculty members are socialized during their graduate school experience to focus primarily on research while they receive little instruction in pedagogy (Atkinson 2001, DeCesare 2003, and Rau and Baker 1989). This lack of pedagogical training in higher education appears to hinder the development of a common culture that promotes pedagogical discourse among faculty members and a shared understanding of how to effectively teach sociology courses. In this study, faculty members echoed this sentiment by noting that a lot of faculty members "do not take great teaching nearly as seriously as they think about their research." One DO group faculty member discussed her disapproval of higher education institutions placing a greater value on research, stating; "I hate that [research outweighs teaching in most institutions]. I think they should at least be equal." Future research could explore how faculty members are able to develop an understanding of their non-research related duties, such as teaching, when a lack of discourse among colleagues is present. Further, might an emphasis on faculty autonomy serve as a potential barrier for developing a common culture among higher education sociology faculty members that values pedagogy as much as research?

In this study, participants described how faculty members may be encouraged to "do your own thing," "to figure out what's good for you," and how "there is very little interference with other people's teaching methods...people mind their own business." This emphasis on faculty autonomy, and the potential norm of 'minding your own business,' is an excellent topic for future research in order to better understand whether or not this influences faculty members' lack of discussion of pedagogy or pedagogical tools. 
For example, if departments or institutions encourage faculty members to 'do their own thing,' might this unintentionally encourage a lack of faculty collaboration regarding pedagogy or pedagogical tools? Further, if faculty members are "assumed" to have the "professionalism to teach their courses," but did not complete a teaching course, could this lead to a lack of discussion with colleagues of pedagogical tools due to a potential fear of not being viewed as a teaching professional? One of the faculty members in this study spoke of this issue when seeking to explain the lack of sharing of pedagogical ideas amongst faculty members. She stated;

“Sometimes people's egos might get in the way of seeing another faculty's pedagogy as impressive, even if they themselves might be interested in the pedagogy...it's like "I don't need that because I'm awesome" and I have to think I'm awesome and portray the image of awesome because we are in a competitive environment"

If faculty members are assumed to possess the professionalism to teach their courses and are encouraged to "do their own thing," might this unintentionally create a competitive environment and prevent the sharing of pedagogical tools? Further research is clearly needed to answer these types of questions. ${ }^{67}$

How Pedagogical Discussions May Be Encouraged Among Faculty Members

Earlier in this chapter, I offered suggestions for increasing the discussion of pedagogical tools among sociology faculty members as well as in graduate teaching

${ }^{67}$ For further illustration of how faculty autonomy (and other barriers identified in this study) may potentially affect a lack of discussion and knowledge of pedagogical tools among faculty members, please see the figure in Appendix I. 
courses. While these suggestions are speculative in nature, future research is required in order to illuminate the best practices for increasing discussion of pedagogical tools among sociology faculty members and in future teaching courses. Based on the findings from this study, it appears that increasing faculty discussion of pedagogical tools can be conducive for; 1) increasing faculty knowledge of pedagogical tools, 2) increasing the legitimization of lesser-known pedagogical tools, and 3) increasing students' levels of engagement in higher education sociology courses by using teaching techniques that are believed by one's colleagues to be effective tools in a specific institution. If faculty members can discuss and learn from their colleagues about which pedagogical tools work best for certain students in certain courses at certain higher education institutions, the overall quality of faculty teaching should improve. Obvious positive benefits for faculty members is that through using pedagogical tools that improve student engagement in learning, they then may be viewed as more competent teachers by their students and receive higher teaching evaluations. ${ }^{68}$ While these potential benefits may be speculative in nature, future research can identify the positive outcomes that are associated with greater faculty discussion of pedagogical tools, and provide greater insight for how the discussion of pedagogical tools may be encouraged among faculty members as well as in completed teaching courses.

68 This was discussed by many faculty members as being important for gaining tenure. 


\section{Conclusion}

I posed two questions in the introduction of this thesis. First, "do higher education sociology faculty members and departments view student engagement as an important precursor to student learning?" Second, "do higher education sociology faculty members and departments view the use of music as an illegitimate pedagogical tool?" In this study, matched-pairs of sociology faculty members by department reported generally believing that student engagement is an important precursor to student learning. Further, sociology faculty participants reported generally viewing the use of music as a potentially beneficial pedagogical tool, regardless of whether they personally used music in their courses.

I have shown that the findings from this study are informative for knowledge, practice, theory, and future research. I have also explained how the findings from this study are informative for illuminating sociology faculty members' perceptions of the potential barriers associated with the use of music as a pedagogical tool as they exist at multiple structural levels. At the individual level, sociology faculty may lack the knowledge to effectively incorporate music into their courses. At the departmental level, a lack of discussion of pedagogical tools among faculty members and in sociology graduate teaching courses may influence faculty members' knowledge and choices about whether or not to use music as a pedagogical tool in their own courses. At an institutional level, failing technology in the classrooms may inhibit faculty members from incorporating music into their sociology courses. And finally, at a macro-level, unclear 
national and international copyright laws may affect higher education sociology faculty's decisions to use music as a pedagogical tool in their classrooms.

It is my hope for the future that sociology departments will include more extensive discussions of the strengths and costs associated with specific pedagogical tools in their graduate teaching courses, as well as provide support and encouragement for faculty members to discuss pedagogical tools and approaches with their colleagues. Through pedagogical discussions, sociology faculty members may increase their knowledge of available pedagogical tools and make better-informed decisions regarding the teaching techniques they choose to implement in their own courses for the betterment of student engagement and learning. This may lead to less students "frequently feeling bored" in their courses, and the utilization of effective pedagogy to "receive the time, effort, and creativity it requires" in order to avoid "a culture of intellectual sloth" (Rau and Baker 1989:171). This is my hope, and is something I will promote as a future higher education sociology faculty member. 


\section{References}

Albers, Benjamin D. and Rebecca Bach. 2003. "Rockin' Soc: Using Popular Music to Introduce Sociological Concepts.” Teaching Sociology 31(2):237-245.

Ahlkvist, Jarl A. 1999. "Music and Cultural Analysis in the Classroom: Introducing Sociology Through Heavy Metal.” Teaching Sociology 27:126-144.

Ahlkvist, Jarl A. 2001. "Sound and Vision: Using Progressive Rock to Teach Social Theory." Teaching Sociology 29(4):471-482.

Andrews, T.M., M.J. Leonard, C.A. Colgrove, and S.T. Kalinowski. 2011. "Active Learning Not Associated with Student Learning in a Random Sample of College Biology Courses." CBE--Life Sciences Education 10:394-405.

Bean, John C. 2011. Engaging Ideas: The Professor's Guide to Integrating Writing, Critical Thinking, and Active Learning in the Classroom. San Francisco: Jossey-Bass.

Bach, Rebecca and Benjamin Albers. 2010. "Rockin' Gender: Using Popular Music to Teach Gender." Class Activity published in TRAILS: Teaching Resources and Innovations Library for Sociology. Originally published 2005 in Sociological Concepts and Gender, edited by M. C. Stalp. Washington DC: American Sociological Association. (http://trails.asanet.org)

Berk, R. A. 2008. "Music and music technology in college teaching: Classical to hip hop across the curriculum." International Journal of Technology in Teaching and Learning, $4(1): 45-67$.

Bligh, D.A. 2000. What's the Use of Lectures? San Francisco: Jossey-Bass.

Bloom, Allan. 1987. The Closing of the American Mind: How Higher Education Has Failed Democracy and Impoverished the Souls of Today's Students. New York: Simon \& Schuster.

Boeije, Hennie. 2002. "A Purposeful Approach to the Constant Comparative Method in the Analysis of Qualitative Interviews." Quality \& Quantity 36:391-409.

Boggs, Carol J. 1993. "Popular Music: Classroom Applications" Pp.18-21 in Songware II. ASA Teaching Resources Center: Washington DC.

Bowman, John R. 1993. "The Sounds of Social Change.” Pp.22-23 in Songware II. ASA Teaching Resources Center: Washington DC. 
Bransford, J.D., Brown, A.L., and Cocking, R.R. 2000. How People Learn: Brain, Mind, Experience, and School. Expanded edition. Committee on Developments in the Science of Learning. Washington, D.C.: National Academy Press.

Burns, Thomas J. and Theresa A. Martinez. 1993. "Pedagogy and Popular Music" Humboldt Journal of Social Relations 19:117-29.

Chalmers, Denise. 2011. "Progress and challenges to the recognition and reward of the Scholarship of Teaching in higher education." Higher Education Research and Development. 30(1):25-38.

Charmaz, Kathy. 2006. Constructing Grounded Theory: A Practical Guide Through Qualitative Analysis. London: Sage Publications.

Cooper, B. Lee. 1982. Images of American Society in Popular Music: A Guide to Reflective Teaching. Chicago: Nelson-Hall.

Cooper, B. Lee and Rebecca A. Condon. 2004. The Popular Music Teaching Handbook: An Educator's Guide to Music-Related Print Resources. Westport, CT: Libraries Unlimited.

D’Silva, Icy. 2010. “Active Learning.” Journal of Education Administration and Policy Studies. 2(6):77-82.

Dean, Paul. 2012. "A Semester of Sociological Theory in Music." Sociology Source. Retrieved February 25, 2012, from http://www.thesociologicalcinema.com/4/post/2011/12/a-semester-of-sociologicaltheory-in-music.html\#comments

DeCesare, Michael. 2003. "The Impact of Sociological Orientation on Pedagogy: A Reconsideration of Teacher Training in Sociology Ph.D. Departments." The American Sociologist 34(3):73-96.

Dorn, Dean S. 1989. "Simulation Games: One More Tool on the Pedagogical Shelf." Teaching Sociology 17(1):1-18.

Elterman, Howard. 1983. "Using Popular Songs to Teach Sociology." Teaching Sociology 10(4):529-538.

Fredericks, Jennifer A., Phyllis C. Blumenfeld, and Alison H. Paris. 2004. "School Engagement: Potential of the Concept, State of the Evidence. " Review of Educational Research 74(1):59-109. 
Greene, Ross W. 2005. The Explosive Child: A New Approach for Understanding and Parenting Easily Frustrated, Chronically Inflexible Children. New York: Harper Collins.

Groce, Stephen B. 1992. "Teaching the Sociology of Popular Music with the Help of Feature Films: A Selected and Annotated Videography." Teaching Sociology 20(1):8084.

Hake, R. 1998. "Interactive-Engagement vs. Traditional Methods: A Six-ThousandStudent Survey of Mechanics Test Data for Introductory Physics Courses." American Journal of Physics 66(1):64.

Hartley, J. and Davies, I. 1978. "Note Taking: A Critical Review." Programmed Learning and Educational Technology 15:207-224.

Higher Education Research Institute. 2011. The American Freshman: National Norms for Fall 2011. University of California, Los Angeles. Retrieved April 5, 2012 from http://gseis.ucla.edu/heri/publications-brp.php

Higher Education Research Institute. 1999. The American Freshman: National Norms for Fall 1999. University of California, Los Angeles. Retrieved April 5, 2012 from http://gseis.ucla.edu/heri/publications-brp.php

Hill, Andrea, Tammi Arford, Amy Lubitow, and Leandra M. Smollin. 2012. "'I'm Ambivalent about it": The Dilemmas of PowerPoint." Teaching Sociology 40(3):242256.

Hillocks, G. 1986. Research on Written Composition: New Directions for Teaching. Urbana, IL: ERIC Clearing House on Reading and Communication Skills and the National Conference on Research in English.

Hillocks, G., Kahn, E.H., and Johannessen, L.R. 1983. "Teaching Defining Strategies as a Mode of Inquiry." Research in the Teaching of English 17(3):275-284

Hinds-Aldrich, Matt. 2012. "Teaching Theory Analogically: Using Music to Explain Criminological Theory." Journal of Criminal Justice Education 23(4):481-499.

Hraba, Joseph, Edward Powers, William Woodman, and Martin Miller. 1980. "Social Change through Photographs and Music: A Qualitative Method for Teaching." Qualitative Sociology 3(2):123-135.

Keskin, Funda. 2011. "Using Songs As Audio Materials In Teaching Turkish As A Foreign Language." The Turkish Online Journal of Educational Technology 10(4):378383. 
Levin, John S. and Genevieve G. Shaker. 2011. "The Hybrid and Dualistic Identity of Full-Time Non-Tenure-Track Faculty." American Behavioral Scientist. 55:1461-1484.

Macheski, Ginger E., Kathleen S. Lowney, Jan Buhrmann, and Melanie E.L. Bush. 2008. "Overcoming Student Disengagement and Anxiety in Theory, Methods, and Statistics Courses by Building a Community of Learners." Teaching Sociology 36:42-48.

Mackenzie, Noella and Sally Knipe. 2006. "Research dilemmas: Paradigms, methods, and methodology." Issues In Educational Research 16(2):193-205.

Maier, Theodore J. 2013. "Music, Anticipatory Sets, and Learning: How to Make Your Students Sit Up and Listen before the Lesson even Begins." NISOD Innovation Abstracts $35(2)$.

Martinez, Theresa A. 1993. "Teaching Sociology with Popular Culture: Classroom Experiences With Music.” Pp. 61-73 in Songware II. ASA Teaching Resources Center: Washington DC.

Martinez, Theresa A. 1994. "Popular Music in the Classroom: Teaching Race, Class, and Gender With Popular Culture." Teaching Sociology 22:260-265.

Martinez, Theresa A. 1995. "Where Popular Culture Meets Deviant Behavior: Classroom Experiences with Music.” Teaching Sociology 23(4):413-418.

Martinez, Theresa A. 1998. "Race an Popular Culture: Teaching African American Leadership Styles through Popular Music.” Teaching Sociology 26(3):207-214.

Mead, George Herbert. 1964 [1934]. On Social Psychology, edited by Anselm Strauss. Chicago: University of Chicago Press.

Mills, C.W. 1959. The Sociological Imagination. New York: Oxford University Press.

Munkres, Susan. 2010. "Music that Challenges the Gender System: A "Real Life" Assignment." Assignment published in TRAILS: Teaching Resources and Innovations Library for Sociology. Originally published 2005 in Sociological Concepts and Gender, edited by M. C. Stalp. Washington DC: American Sociological Association. (http://trails.asanet.org)

Neisser, Ulric. 1976. Cognition and Reality: Principles and Implications of Cognitive Psychology. San Francisco: W.H. Freeman and Company.

Newmann, F.M., G.G. Wehlage, and S.D. Lamborn. 1992. "The significance and sources of student engagement." Pp. 11-39 in Student Engagement and achievement in American secondary schools. New York: Teachers College Press. 
Norman, D.A. 1980. "What Goes On in the Mind of the Learner." In W.J. McKeachie (ed.), Learning, Cognition, and College Teaching. New Directions for Teaching and Learning, no. 2. San Francisco: Jossey-Bass.

Palmer, Nathan. 2011. "iTeach with iTunes How About You?" Sociology Source. Retrieved February 25, 2012, from http://www.sociologysource.com/home/2011/4/4/iteach-with-itunes-how-about-you.html

Pekrun, Reinhard, Thomas Goetz, Wolfram Titz, and Raymond P. Perry. 2002.

"Academic Emotions in Students' Self-Regulated Learning and Achievement: A Program of Qualitative and Quantitative Research." Educational Psychologist 37(2):91-105.

Pescosolido, Bernice A. 1990. "Teaching Sociology through film: Theorectical Perspectives and Practical Tool." Teaching Sociology 18(3):337-346.

Pichaske, David R. 1999. "Poetry, Pedagogy, and Popular Music: Renegade Reflections." Popular Music and Society 23(4):83-103.

Prince, Michael. 2004. "Does Active Learning Work? A Review of the Research." Journal of Engineering Education 93(3):223-231.

Ragin, Charles C. and Lisa M. Amoroso. Constructing Social Research. 2nd ed. Thousand Oaks, CA: Pine Forge Press.

Reuter, Monika. 1993. "People retain information better when they can sing it - student evaluations." Pp.74-86 in Songware II. ASA Teaching Resources Center: Washington DC.

Scanlan, Stephen J. and Seth L. Feinberg. 2000. "The Cartoon Society: Using "The Simpsons" to Teach and Learn Sociology" Teaching Sociology 28(2):127-139.

Schacht, Steven P. 1990. "Statistics Textbooks: Pedagogical Tools or Impediments to Learning?" Teaching Sociology 18(3):390-396.

Schneider, Christopher. 2011 "Using the MP3 to Learn Selections of Marx's Capital." Assignment published in TRAILS: Teaching Resources and Innovations Library for Sociology. Washington DC: American Sociological Association. (http://trails.asanet.org)

Shade, William L. and Joann P. Paine. 1975. "Simulation in the Classroom." Teaching Political Science 3:83-89

Shae, Brent Mack. 1993. "A Content Analysis of the Lyrics of American Popular Music from 1967 to 1970” Pp.91-109 in Songware II. ASA Teaching Resources Center: Washington DC. 
Schatzki, T. 2002. The site of the social: A philosophical account of the constitution of social life and change. University Park, PA: University of Pennsylvania Press.

Terhune, T. 1997. "Pop Songs." The English Connection 1:8-12.

Thomas, Gary. 2009. How to do your Research Project: A Guide For Students In Education \& Applied Social Sciences. London: Sage Publications.

Thirolf, Kathryn Q. 2012. "The Faculty Identitites of Community College Adjuncts Teaching in the Humanities: A Discourse Analysis Study." Community College Journal of Research and Practice 36(4):269-278.

Walczak, David A., Janet Merrill Alger, and Monika Reuter. 1989. SONGWARE: Using Popular Music in Teaching Sociology Washington D.C., ASA Teaching Resources Center.

Walczak, David and Monika Reuter. 1993. "Using Song Lyrics as Text.” Pp.120- in Songware II. ASA Teaching Resources Center: Washington DC.

Walczak, David and Monika Reuter. 1994. "Using Popular Music To Teach Sociology: An Evaluation By Students.” Teaching Sociology 22:266-269.

Walczak, David. 1993. "Using Popular Music to Teach Conflict Theory" Pp.110-119 in Songware II. ASA Teaching Resources Center: Washington DC.

Wankat, P. 2002. The Effective Efficient Professor: Teaching, Scholarship and Service. Boston: Allyn and Bacon.

Zull, James E. 2002. The Art of Changing the Brain: Enriching the Practice of Teaching By Exploring the Biology of Learning. Sterling, Virginia: Stylus Publishing.

Zyngier, David. 2004. "Doing education not doing time. Engaging Pedagogies and Pedagogues - what does student engagement look like in action?" International Education Research Conference, Melbourne, Australia. 


\section{Appendix A: Recruitment Email I}

Dear faculty name,

You are invited to participate in a study exploring your experiences with using music (i.e. songs and lyrics) as a pedagogical tool in higher education sociology courses. This study will be conducted by Jerry Loveless, a Graduate Student in the Portland State University Sociology Department, and supervised by Dr. Peter Collier - Department of Sociology. The purpose of this study is to explore higher education sociology faculty's views regarding the use of music as a pedagogical technique and of its relation to student engagement in the sociology classroom. Your participation will contribute to further understanding how music and student engagement are viewed by sociology faculty, and findings could be used to support positive changes in higher education sociology courses.

If you are interested in participating, please reply via email to Jerry Loveless at glove2@pdx.edu, or by phone at the given number below. If you are not interested, please reply to let me know you would prefer not to be contacted again. If I don't hear from you, I will follow up via email or by phone to answer any questions and invite you to participate.

Best regards, Jerry

Jerry Loveless

Portland State University

Graduate School of Sociology

1721 SW Broadway \#217W

Portland, OR 97207

Phone: 503-725-5820

Email: glove2@pdx.edu 


\section{Appendix B: Recruitment Email II}

Dear faculty name,

You are invited (or, have been referred by Dr. "Jane Doe") to participate in a study exploring your experiences with pedagogical tools and student engagement in higher education sociology courses. This study will be conducted by Jerry Loveless, a Graduate Student in the Portland State University Sociology Department, and supervised by Dr. Peter Collier - Department of Sociology. The purpose of this study is to explore higher education sociology faculty perspectives regarding certain pedagogical techniques and of their relation with student engagement in sociology courses. Your participation will contribute to further understanding how certain pedagogical tools and student engagement are viewed by higher education sociology faculty. Findings from this study could be used to support positive changes in higher education sociology courses.

If you are interested in participating, please reply via email to Jerry Loveless at glove2@pdx.edu, or by phone at the given number below. If you are not interested, please reply to let me know you would prefer not to be contacted again. If I don't hear from you, I will follow up via email or by phone to answer any questions and invite you to participate.

Best regards, Jerry

Jerry Loveless

Portland State University

Graduate School of Sociology

1721 SW Broadway \#217W

Portland, OR 97207

Phone: 503-725-5820

Email: glove2@pdx.edu 


\section{Appendix C: Recruitment Telephone Script I}

Hello faculty name,

This is Jerry Loveless calling from Portland State University to invite you to participate in a thesis study exploring the use of music as a pedagogical tool and student engagement in higher education sociology courses.

Participation would include completing a short interview (approximately 60 minutes in length) via Skype or by telephone, and would include a small incentive for your time.

Please give a call, or email, to setup a suitable date and time for your interview. Your participation is very important toward the completion of this study.

Thank you, and have a great day! 


\section{Appendix D: Recruitment Telephone Script II}

Hello faculty name,

This is Jerry Loveless calling from Portland State University to invite you to participate in a thesis study exploring pedagogical tools and student engagement in higher education sociology courses.

Participation would include completing a short interview (approximately 60 minutes in length) via Skype or by telephone, and would include a small incentive for your time.

Please give a call, or email, to setup a suitable date and time for your interview. Your participation is very important toward the completion of this study.

Thank you, and have a great day! 


\section{Appendix E: Informed Consent Document}

\section{Informed Consent}

Pedagogical Tools and Student Engagement in The Higher Education Sociology Course: Exploring Faculty Perspectives

Congratulations, you are invited to participate in a research study conducted by Jerry Loveless - a Graduate Student in the Portland State University Sociology Department. The researcher hopes to learn more about the views of higher education sociology instructors regarding certain pedagogical tools and student engagement. This study is being conducted in partial fulfillment of the requirements for a Master's degree, and will be supervised by Dr. Peter Collier of Portland State University, Department of Sociology.

What Will I Have To Do?

If you decide to participate, you will be asked to meet with the researcher for up-to 90 minutes discussing these general topics:

- Your experiences leading up to becoming a sociology professor

- Your experiences working in higher education as a sociology professor

- Your experiences with pedagogy, student engagement, and structural barriers

- And, your understanding of how certain pedagogical tools impact learning

How Will Data Be Collected?

If you decide to participate, you will be asked to complete a private one-on-one interview either in-person, via Skype (i.e. a free online video conferencing service), or by telephone. These interviews will include the recording of audio, with full transcriptions of the conversation being completed for future data analysis.

\section{Are There Any Risks?}

Participation in this study has the possibility of causing inconvenience and/or mild psychological discomfort in the form of anxiety, stress, depression, embarrassment, anger, and/or guilt while sharing your personal experiences. Your participation is voluntary throughout, you may refuse to answer any of the interview questions and/or withdraw your participation at any time without negative consequences. It is also possible that others may become aware of your participation in this study, and your responses to the questions asked during the interview. To overcome this, no identifying information will be recorded during the interview (e.g. your name, address, or location of the interview), and the recorded audio will only be identifiable by an assigned case number. Original recordings will be disposed of after they have been fully transcribed in a manner that discards all potentially identifying information. Further, all discussions during the interview will be kept private and confidential, with all data being kept in a 
locked filing cabinet or on a password protected hard-drive - also kept under lock and key.

What Are The Benefits?

The information gathered in this study has the potential to increase the knowledge regarding how different pedagogical tools are viewed by higher education sociology faculty as they relate to student engagement. The attained information could be used to support positive changes in higher education sociology courses; potentially leading to an increase in student engagement and retention rates. As a small token of the researcher's appreciation for your time, a gift will be presented at the time of the interview.

\section{Any Questions?}

If you have any concerns or problems regarding your participation in this study, or your rights as a research participant, please contact the Human Subjects Research Review Committee, Office of Research and Sponsored Projects, Market Center Build 1600 SW $4^{\text {th }}$ Avenue suite $620,6^{\text {th }}$ Floor, Portland State University, 503-725-4288. If you have any questions about the study itself, please contact Jerry Loveless at the Department of Sociology, 217W Cramer Hall, Portland State University, (503) 725-5820, or by email: glove2@pdx.edu.

Your participation is voluntary. You do not have to take part in this study. Your verbal consent (given at the beginning of the interview) indicates that you have read and understand the above information, and agree to take part in this study. Please understand that you may withdraw your consent at any time without penalty, and, by giving your verbal consent, you are not waiving any legal claims, rights or remedies. The researcher will provide you a copy of this informed consent via email for your records.

Best regards,

Jerry

Jerry Loveless

Portland State University

Graduate School of Sociology

(503) 725-5820

glove2@pdx.edu 


\section{Appendix F: Interview Guide I}

\section{Interview guide I}

1) Have you read the informed consent document?

2) Do you willingly give your consent to be interviewed for this study?

3) What is your current position? Please include your title, department, and the name of your institution. (probe: employment status - full time? tenure-track?)

4) How long have you been teaching at this institution?

5) Which courses do you currently teach?

\section{Background/Education}

1) Can you tell me a little bit about your graduate school experience? (probe: where/when attended?)

2) What types of pedagogical approaches were used in your graduate courses? (probe: for each unknown approach, can you give an example?)

3) What types of pedagogical tools did your professors use?

4) Did you complete any courses on effective approaches for teaching at the collegiate level?

- Yes - Tell me about some of the approaches to pedagogy they covered in this course? Did the course(s) cover strengths and weaknesses of particular pedagogical tools? Which tools did you find engaging? Tell me more. Which tools seemed potentially useful?

- No - Tell me about some of the pedagogical tools utilized in your graduate courses. Which tools did you find engaging? Tell me more. Which tools seemed potentially useful?

\section{Teaching Experience and Music as a Pedagogical Tool}

1) Do you feel student engagement leads to increased learning? (probe: can you please give an example from your experience?)

2) What types of pedagogical tools do you use in the courses you teach? (probe: how long have you been using music as a pedagogical tool?) 
3) What led to your decision to begin using music as a pedagogical tool in your courses?

4) In what ways do you, or have you, used music in your courses?

5) How do you feel the use of music impacts learning? (probe: from your own experience as a student, do you think music may have increased your level of engagement with sociology courses? How so?)

6) What do you see as the potential benefits of using music in your courses? (probe: do you feel music has the potential to increase student engagement? Tell me more, please explain.)

7) What do you see as the potential costs of using music in your courses? (probe: Tell me more, please explain.)

8) Approximately what percentage of sociology faculty at your school would you guesstimate use music as a pedagogical tool in their college courses? (probe: please explain)

9) Approximately what percentage of sociology faculty at universities and colleges in the U.S. would you guesstimate use music as a pedagogical tool in their courses? (probe: please explain, can you speculate as to why only ___ $\%$ use music as a pedagogical tool?)

10) Are you likely to continue using music as a pedagogical tool in your sociology courses in the future? (probe: please explain)

\section{Structural Barriers and Other Pedagogical Tools}

1) What types of pedagogical tools are used by other faculty members in your department? (probe: how do you feel these techniques impact learning?)

2) How do you think your colleagues view the use of music as a pedagogical tool?

3) Would you say that some pedagogical tools are encouraged or discouraged by your department? How about by your institution? (probe: for each mentioned, can you give me an example?)

4) What types of structural barriers have you encountered when trying to utilize music as a pedagogical tool in your courses? (probe: for each mentioned, can you give me an example?) 
5) How have you, or other faculty who use music in their courses, chosen to deal with these barriers?

Closing: 'we are nearing the end of the interview, but beforehand I would like to ask you a few simple demographic questions:'

- In which decade were you born?

- What was your mother's occupation when you were a teenager (12-19)? What was your father's occupation when you were a teenager?

- Do you have children?

- Which race or ethnicity do you identify with?

1) Which musicians do you currently enjoy listening to? (probe: How often do you purchase music? How often do you attend concerts?)

2) Do you play any musical instruments?

3) Do you have any questions for me?

4) Would you like to receive a short summary of the findings from this study?

5) Can I contact you if further clarity is needed?

6) Are there any questions that I should have included in this interview?

7) Would you be willing to recommend someone from your department that may be demographically similar to yourself, and who is unlikely to use music in their courses, to complete an interview?

- If Yes: do you mind if I use your name as the source of referral when contacting them?

- Could you please keep the specific focus on music as a pedagogical tool confidential until after I complete the interview with your colleague? I will send you an email upon completion of the interview.

8) Do you know of any other higher education sociology professors who use music in their courses that may be interested in participating in this study?

9) Is there anything else you would like to mention? 
'Thank you greatly for your participation! For your valuable time, I would love to give you the gift of music. I'm emailing you a \$10 iTunes gift card so you can "keep on rockin' in the free world!" Thanks again.' 


\section{Appendix G: Interview Guide II}

\section{Interview guide II}

1) Have you read the informed consent document?

2) Do you willingly give your consent to be interviewed for this study?

3) What is your current position? Please include your title, department, and the name of your institution. (probe: employment status - full time? tenure-track?)

4) How long have you been teaching at this institution?

5) Which courses do you currently teach?

\section{Background/Education}

5) Can you tell me a little bit about your graduate school experience? (probe: where/when attended?)

6) What types of pedagogical approaches were used in your graduate courses? (probe: for each unknown approach, can you give an example?)

7) What types of pedagogical tools did your professors use?

8) Did you complete any courses on effective approaches for teaching at the collegiate level?

- Yes - Tell me about some of the approaches to pedagogy they covered in this course? Did the course(s) cover strengths and weaknesses of particular pedagogical tools? Which tools did you find engaging? Tell me more. Which tools seemed potentially useful?

- No - Tell me about some of the pedagogical tools utilized in your graduate courses. Which tools did you find engaging? Tell me more. Which tools seemed potentially useful?

\section{Teaching Experience and Pedagogical Tools}

1) Do you feel student engagement leads to increased learning? (probe: can you please give an example from your experience?)

2) What types of pedagogical tools do you use in the courses you teach? (probe: do you have a preferred pedagogical tool? How long have you been using 
3) What led to your decision to begin using in your courses?

4) In what ways do you, or have you, used in your courses?

5) How do you feel impacts learning? (probe: from your own experience as a student, do you think with sociology courses? How so?)

6) What do you see as the potential benefits of using ? (probe: do you feel has the potential to increase student engagement? Tell me more, please explain.)

7) What do you see as the potential costs of using in your courses? (probe: Tell me more, please explain.)

8) Are you likely to continue using as a pedagogical tool in your sociology courses in the future? (probe: please explain)

\section{Structural Barriers and Other Pedagogical Tools}

10) What types of pedagogical tools are used by other faculty members in your department? (probe: how do you feel these techniques impact learning?)

11) How do you think your colleagues view the use of as a pedagogical tool?

12) Would you say that some pedagogical tools are encouraged or discouraged by your department? How about by your institution? (probe: for each mentioned, can you give me an example?)

13) What types of structural barriers have you encountered when trying to utilize as a pedagogical tool in your courses? (probe: for each mentioned, can you give me an example?)

14) How have you, or other faculty who use in their courses, chosen to deal with these barriers?

\section{Music as a Pedagogical Tool}

1) Have you known any sociology faculty who have used music as a pedagogical tool? (for each: in what ways have they used music, please explain?) 
2) How do you feel the use of music in college sociology courses might impact learning?

3) Do you feel music can be an effective pedagogical tool for promoting student engagement in college sociology courses? (probe: costs/benefits/structural barriers?)

4) Approximately what percentage of sociology faculty at your school would you guesstimate use music as a pedagogical tool in their college courses? (probe: please explain)

5) Approximately what percentage of sociology faculty at universities and colleges in the U.S. would you guesstimate use music as a pedagogical tool in their courses? (probe: please explain, can you speculate as to why only ___ $\% \underline{\text { use }}$ music as a pedagogical tool?)

6) Would you be likely to use music as a pedagogical tool in your sociology courses in the future? (probe: please explain)

Closing: 'we are nearing the end of the interview, but beforehand I would like to ask you a few simple demographic questions:'

- In which decade were you born?

- What was your mother's occupation when you were a teenager (12-19)?

What was your father's occupation when you were a teenager?

- Do you have children?

- Which race or ethnicity do you identify with?

1) Which musicians do you currently enjoy listening to? (probe: How often do you purchase music? How often do you attend concerts?)

2) Do you play any musical instruments?

3) Do you have any questions for me?

4) Would you like to receive a short summary of the findings from this study?

5) Can I contact you if further clarity is needed?

15) Are there any questions that I should have included in this interview? 
7) Do you know of any other higher education sociology professors who use music in their courses that may be interested in participating in this study?

8) Is there anything else you would like to mention?

'Thank you greatly for your participation! For your valuable time, I would love to give you the gift of music. I'm emailing you a \$10 iTunes gift card so you can "keep on rockin' in the free world!' Thanks again.' 


\section{Appendix H: Codebook}

\section{$\underline{\text { Codebook }}$}

1000. Pedagogical Tools

1000. Used by professors during graduate experience

1300. Discussed in completed teaching course

1400. Used by colleagues

1600. Pedagogical Tools used in own teaching experience

2000. Pedagogical Approaches

2000. Used by professors during graduate experience

2300. Discussed in completed teaching course

2600. Used in own teaching experience

3000. Feels Student Engagement Leads to Increased Learning

4000. Preferred Pedagogical Tool

4000. Benefits

4300. Costs

4600. Potential Structural Barriers

5000. How Preferred Pedagogical Tool is Viewed by Colleagues

5000. Positive

5300. Negative

5600. Neutral

6000. Music as a Pedagogical Tool

6000. Benefits

6300. Costs

6600. Potential Structural Barriers

7000. How The Use of Music is Potentially Viewed by Colleagues 7000. Positive

7300. Negative

7600. Neutral

8000. Likelihood of Future Usage of Music as a Pedagogical Tool

9000. Other

9100. Lecture

9100 Negatives of straight lecture 
9150 Positives of straight lecture

9200. Pedagogy/Pedagogical Tools not discussed often

9250 more focus on research

9300. Won Teaching Awards?

9400. Pedagogical Tools Encouraged/Discouraged

9400. Encouraged by Department

9420. Discouraged by Department

9440. Encouraged by Institution

9460. Discouraged by Institution

9500. Influenced Decision to Use Preferred Pedagogical Tool/Music

9600. Use of Music Viewed as Common in U.S. (over 50\%)?

9650 Guesstimated Percentage of Soc. Faculty that Use Music in Department

9670 Guesstimated Percentage of Soc. Faculty that Use Music in U.S.

9690 Guesstimated Percentage of Soc. Faculty that Use Music in U.S. and Canada

9700. Other Potential Structural Barriers Mentioned

9800. Overcoming Structural Barriers

9800. Associated with Using Music as a Pedagogical Tool

9850. Associated with Preferred Pedagogical Tool

9890. Associated with Other Pedagogical Tools

99999. Indecipherable 
Appendix I: Figure of Potential Barriers to Inform Future Research

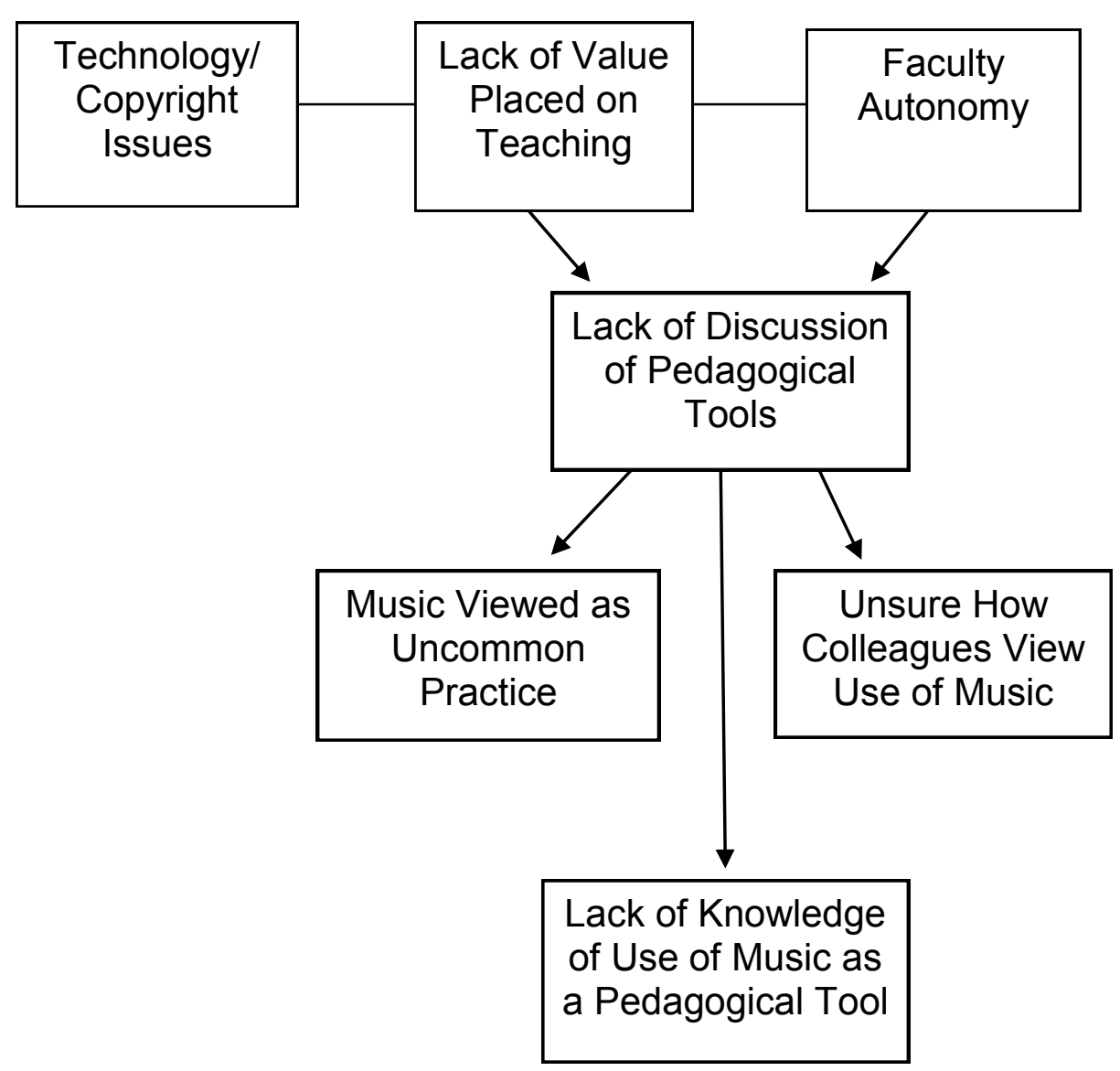




\title{
Appendix J: Human Subjects Approval
}

\section{Portland State University HSRRC Memorandum}

\author{
To: $\quad$ Gerald Loveless
}

From: Mary Oschwald, Chair, HSRRC 2012

Date: June 7, 2012

Re: Your HSRRC application titled, "Exploring Faculty Perspectives on Pedagogical Approaches to Promoting Student Engagement: The Use of Music in Higher Education Sociology Courses” (HSRRC Proposal \#122195)

In accordance with your request, the Human Subjects Research Review Committee has reviewed your proposal referenced above for compliance with DHHS policies and regulations covering the protection of human subjects. The committee is satisfied that your provisions for protecting the rights and welfare of all subjects participating in the research are adequate, and your project is approved.

Please note the following requirements:

Changes to Protocol: Any changes in the proposed study, whether to procedures, survey instruments, consent forms or cover letters, must be outlined and submitted to the Chair of the HSRRC immediately. The proposed changes cannot be implemented before they have been reviewed and approved by the Committee.

Continuing Review: This approval will expire 6/07/2013, one year from the approval date. It is the investigator's responsibility to ensure that a Continuing Review Report (available in ORSP) of the status of the project is submitted to the HSRRC approximately two months before the expiration date, and that approval of the study is kept current.

Adverse Reactions: If any adverse reactions occur as a result of this study, you are required to notify the Chair of the HSRRC immediately. If the problem is serious, approval may be withdrawn pending an investigation by the Committee.

Completion of Study: Please notify the Chair of the Human Subjects Research Review Committee (campus mail code ORSP) as soon as your research has been completed. Study records, including protocols and signed consent forms for each participant, must be kept by the investigator in a secure location for three years following completion of the study. 
If you have questions or concerns, please contact the HSRRC in the Office of Research and Strategic Partnerships, Market Center Building, Suite 620, 1600 SW Fourth Ave, Portland OR 97207 (503)725-2243.

cc: Ann Stephenson, Pete Collier 